\title{
Dynamics of Transition Regime in Bi-stable Vibration Energy Harvesters
}

\author{
Alwathiqbellah Ibrahim \\ Graduate Research Assistant \\ Department of Mechanical Engineering \\ Binghamton University \\ 4400 Vestal Parkway E. \\ Binghamton, NY 13902 \\ Email: aibrahi4@binghamton.edu
}

\author{
Shahrzad Towfighian \\ Assistant Professor \\ Department of Mechanical Engineering \\ Binghamton University \\ 4400 Vestal Parkway E. \\ Binghamton, NY 13902 \\ Email: stowfigh@binghamton.edu
}

\author{
Mohammad Younis \\ Associate Professor \\ Department of Mechanical Engineering \\ State University of New York at Binghamton \\ 4400 Vestal Parkway E. \\ Binghamton, NY 13902 \\ Email: myounis@binghamton.edu \\ King Abdullah University of Science and Technology \\ 23955-6900 Thuwal, KSA \\ Email: mohammad.younis@kaust.edu.sa
}

\begin{abstract}
Abstract

Vibration energy harvesting can be an effective method for scavenging wasted mechanical energy for use by wireless sensors that have limited battery life. Two major goals in designing energy harvesters are enhancing the power scavenged at low frequency and improving efficiency by increasing the frequency bandwidth. To achieve these goals, we derived a magneto-elastic beam operated at the transition between mono- and bi-stable regions. By improving the mathematical model of the interaction of magnetic force and beam dynamics, we obtained a precise prediction of natural frequencies as the distance of magnets varies. Using the shooting technique for the improved model, we present a fundamental understanding of interesting combined softening and hardening responses that happen at the transition between the two regimes. The transition regime is proposed as the optimal region for energy conversion in terms of frequency bandwidth and output voltage. Using this technique, low frequency vibration energy harvesting at around $17 \mathrm{~Hz}$ was possible. The theoretical results were in good agreement with the experimental results. The target application is to power wildlife bio-logging devices from bird flights that have consistent high power density around $16 \mathrm{~Hz}$ [1].
\end{abstract}

\section{Introduction}

Ambient energy surrounds us in different forms, such as wind, solar, thermal, and mechanical energy. Many of these are considered waste energy from the naturally generated sources. The process of scavenging and storing such energy is known as energy harvesting.

A major source of energy to harvest is the prevalent mechanical vibration present in the environment and wildlife. Vibration energy harvested from these sources can be useful for autonomous powering of wireless sensors [1-7]. With the advancement of sensor technologies, less than $1 \mathrm{~mW}$ of power is sufficient to efficiently run these sensors [8-11]. Such low power consumption inspires the use of alternative energy sources to address the limited life and replacement costs of 
batteries. Vibration energy harvesters are mechanical resonators that oscillate when attached to a vibration source [12-14]. This oscillation is then converted into electric energy using electromagnetics [15-17], electrostatic [18, 19] or piezoelectric [20-37] transducers. The latter type is the most common mechanism because of its high-energy density.

Linear resonators are inefficient because of their narrow frequency bandwidth compared with the broadband ambient vibrations. For higher efficiency, the linear resonator frequency should be altered to match the excitation frequency from the ambient source. Several methods for tuning the resonator frequency with the dominant ambient frequency were explored including the use of magnets [38-45], but these did not change the nature of the narrow bandwidth. To solve the bandwidth problem, several nonlinear resonators have been investigated during the past few years.

Nonlinear systems have higher bandwidth leading to an increase in the efficiency of the harvester. Sources of nonlinearities include magnetic [20,46-59] and mechanical [29,30,60,61] forces. A common magnetoelastic resonator is composed of a cantilever beam with a tip magnet facing the same pole of a stationary magnet. Magnetic nonlinearity, hardening or softening, can be achieved by controlling the distance between two magnets. Increasing the distance will cause the system to have a single stable equilibrium point and hardening behavior will occur, while reducing the distance will cause the system to have two stable equilibrium points and softening behavior will appear.

Several mono-stable energy harvesters in the micro-scale [62-65] or the macro-scale [49] have been developed for broadening bandwidth. Some examples include the use of microbeams under bending and stretching [62, 63], stoppers [64] and nonlinear springs [65].

Using bi-stability to increase the output power and frequency bandwidth was investigated by Erturk et al. [53,66]. Different studies used the same approach to efficiently harvest energy [17, 18,36,45,55,67-70]. Masana and Daqaq [29] showed that the potential well for the bi-stable harvester depends on the excitation levels and achieved larger bandwidth but only at higher excitation levels. A similar design but with added lumped mass was investigated by Sneller et al. [30], in which the added mass reduces the excitation level required for the harvester to oscillate between the two potential wells.

For bi-stable energy harvesters, experiments show the highest energy is produced at the transition from the mono-stable to the bi-stable region [71,72]. However, a deep understanding and analysis of the complicated behavior of the harvester in the transition regime is still absent. This calls for accurate models and analysis tools to be developed and applied. For example, analytical methods such as harmonic balance [57,73] have been used to solve the governing equations of motion. However, to capture more accurate dynamical responses, higher order harmonics should be considered, which makes the analysis very sophisticated. The perturbation methods are also limited to weak nonlinearities [74] and are not capable of capturing combined softening and hardening nonlinear responses [75]. Among the possible numerical techniques for analysis, the most commonly used method of long time integration is computationally inefficient and unable to predict complex bifurcation behaviors. In this study, we have addressed this limitation by using the shooting technique that can capture all the possible bifurcations including the combined softening and hardening behavior in the transition region. This feature allows studying in details the broadening of the frequency bandwidth. The shooting technique updates the initial conditions until it finds the steady state solution of the dynamical system and determines its stability based on the Floquet multipliers. It is more efficient than other numerical methods and, unlike perturbation methods, is not limited to the small nonlinearities or forcing. The presented simulation results are validated by experimental results.

An important aspect of the proposed energy harvester is the low frequency conversion capability. Several structures have been employed to decrease the frequency of vibration for energy harvesting to below $100 \mathrm{~Hz}$, including a zigzag micro structure with a tip mass [76], a composite bi-stable plate [77], and an impact energy harvester [78]. Our results indicate bistable harvesters at the transition regime, which not only generate larger voltage, but also achieve larger bandwidth at a lower frequency (around $17 \mathrm{~Hz}$ ). The transition regime can be determined by tuning the magnetic distance to the distance between the mono- to bi-stable regions. The mentioned characteristics make the transition regime attractive, and hence call for more attention. The variations of the natural frequency and voltage output with the distance between the permanent magnets was explored experimentally [68-70,79]. However, determining accurately determines the fundamental natural frequency of the resonator as a function of distance between two magnets remain missing. Predicting of natural frequency in the transition region is important, which maybe determined using a lumped parameter model [34]. Using a continuous model, we obtain a good agreement between the predicted and measured natural frequencies as the distance between magnets varied. Precise prediction of the dynamic responses and natural frequencies shed light on the underlying mechanism of the transition regime of bi-stable energy harvesters and can be used as a useful tool to design optimal energy harvesters for applications such as self-powered bird tags [1].

The outline of the paper is as follows. Section 2 describes the principle of operation of the resonator and the experimental

VIB-16-1454 . Towfighian . 2 
Copyright (setup. A free vibration model of the system is developed in Section 3. Section 4 expresses the magnetic force. Section 5 , 6 , and 7 elaborates the static analysis, eigenvalue problems and dynamic analysis, respectively. Results and discussions are presented in Section 8 followed by the conclusions in Section 9.

\section{Principle of Operation}

The nonlinear resonator is composed of a piezoelectric cantilever beam with a tip magnet facing the same pole of another magnet (Fig. 1a). To increase the amplitude of the resonator and lower its natural frequency, we use a polymer material for the cantilever beam that is partially covered by piezoelectric laminates. The magnetic force acts as a nonlinear spring that makes the system bi-stable. There have been studies on modeling this bi-stable resonator that mostly focus on lumped parameter modeling [68]. However, lumped modeling is only an approximation for the system's response at frequencies close to its natural frequency. To accurately evaluate the response of the resonator for a wide range of frequencies, we develop a continuous model of the system and determine the response to a harmonic base excitation, $\ddot{z}(t)=A \cos (\Omega t)$, where $\Omega$ is the frequency of excitation and $A$ is the amplitude. In Fig. 1a, $d$ is the horizontal distance between two magnets, $L$ is the length of the beam, $L_{1}$ is the length of the piezoelectric layer on the top and bottom, $W(x, t)$ is the transverse deflection of the beam, $R_{L}$ is the load resistance, and $F_{m a g}$ is the magnetic force. The tip magnet is modeled as a rigid body with mass $M$ and mass moment of inertia $J=\frac{1}{6} M L_{m}^{2}$ with a side length $L_{m}$.

The effect of the distance between magnets on the beam response and potential energy function is illustrated in Fig. 1b. Magnetic force is a function of separation distance and is responsible for creating one more equilibrium points to make a bi-stable system. The corresponding potential energy function is a single well or double well depending on the distance between magnets. For large distances between magnets, the beam oscillates around one stable point, point 1 (mono-stable behavior). At small distances, the system has two potential wells and the beam oscillates between two stable equilibrium points, at points 2 and 3, (bi-stable behavior).

Fig. 2 shows the experimental setup used to test the magnetoelastic energy harvester. The setup consists of a PUMA Spectral Dynamics Analyzer (PSDA) and the resonator attached to a rigid base with a rail to adjust the initial magnet distance. The PSDA sends the signal to the amplifier which amplifies the signal to vibrate the shaker at the specified excitation amplitude for a required range of excitation frequency. The resonator and its base were placed on a shaker to apply the base vibration. A schematic for the experimental setup and connections is shown in Fig. 2.

The materials used include a polymeric beam made of Makrolon polycarbonate with modulus of elasticity of $2.34 \mathrm{GPa}$. The beam is partially covered by a piezoelectric material of lead zirconate titanate (PZT) 5A Navy Type II with modules of elasticity of $66 \mathrm{GPa}$, and it has a piezoelectric voltage constant of $\varepsilon_{31}=-11 \times 10^{-3} \mathrm{Vm} / \mathrm{N}$, at the top and bottom. The cubic magnets have a side length of $8 \mathrm{~mm}$ and magnetic moment of $0.5 A^{2} / \mathrm{m}$. The rest of the parameters are listed in Table 1 . Two accelerometers; PCB Piezotronics 352C22 and 352A24; with sensitivities of $10.28 \mathrm{mV} / \mathrm{g}$ and $98 \mathrm{mV} / \mathrm{g}$ and $0.5 \mathrm{gm}$ and $0.8 \mathrm{gm}$ weights, respectively, were used to measure the output and input acceleration signals. The accelerometer with higher sensitivity was placed on the rigid holder while the accelerometer with lower sensitivity was placed on the tip magnet. Both accelerometers were connected to the acceleration data logger, which was connected to the PSDA. The data logger had many channels to receive the data from the accelerometers and the piezoelectric circuit.

\section{Free Vibration Model}

The energy harvester has two parts: the first is the biomorph with the piezoelectric layer at the top and bottom $(0 \leq$ $\left.x \leq L_{1}\right)$, and the second consists of a polymer layer $\left(L_{1} \leq x \leq L\right)$. By applying Newton's second law and considering the continuity conditions at $L_{1}$, the governing equations for free vibration (no base excitation) of the system and boundary conditions are obtained.

$$
\begin{array}{ll}
\rho A_{c} \ddot{W}_{1}(x, t)+E I_{c} W_{1 x x x x}(x, t)+c \dot{W}_{1}(x, t)-\vartheta V(t)\left[\frac{d \delta(x)}{d x}-\frac{d \delta(x-L)}{d x}\right]=0, & 0 \leq x \leq L_{1} \\
\rho_{p} A_{p} \ddot{W}_{2}(x, t)+E_{p} I_{p} W_{2 x x x x}(x, t)+c \dot{W}_{2}(x, t)=0, & L_{1} \leq x \leq L \\
C_{p} \dot{V}(t)+\left.\vartheta \frac{\partial \dot{W}(x, t)}{\partial x}\right|_{L_{1}}+\frac{V(t)}{R_{L}}=0 &
\end{array}
$$




$$
\begin{aligned}
& W_{1}(0, t)=0, \quad W_{1 x}(0, t)=0 \\
& W_{1}\left(L_{1}, t\right)-W_{2}\left(L_{1}, t\right)=0 \\
& W_{1 x}\left(L_{1}, t\right)-W_{2 x}\left(L_{1}, t\right)=0 \\
& E I_{c} W_{1 x x}\left(L_{1}, t\right)-E_{p} I_{p} W_{2 x x}\left(L_{1}, t\right)=0 \\
& E I_{c} W_{1 x x x}\left(L_{1}, t\right)-E_{p} I_{p} W_{2 x x x}\left(L_{1}, t\right)=0 \\
& E_{p} I_{p} W_{2 x x x}(L, t)-M \ddot{W}_{2}(L, t)-M L_{c} \ddot{W}_{2 x}(L, t)+F_{\text {magy }}-M g=0 \\
& E_{p} I_{p} W_{2 x x}(L, t)+M L_{c} \ddot{W}(L, t)+\left[M L_{c}^{2}+J\right] \ddot{W}_{2 x}(L, t)-L_{c} F_{m a g y}+L_{c} M g=0
\end{aligned}
$$

where $\rho A_{c}$ is the equivalent mass per unit length, for the biomorph part of the beam $\left(\rho_{1} A_{1}+\rho_{p} A_{p}+\rho_{2} A_{2}\right)$. The subscripts $(1,2)$ denote the biomorph and the polymer parts of the beam. $c$ is the damping, $\delta$ is the Dirac delta function, $E_{p}$ and $I_{p}$ are the polymer beam modulus of elasticity and the second moment of inertia, respectively. $V$ and $R_{L}$ are the voltage and resistance load, respectively, $C_{p}=\frac{2 b L \varepsilon_{33}^{\varepsilon_{x x}}}{h_{1}}$ is the capacitance through two piezoelectric layers connected in parallel. $E I_{c}$ and $\vartheta$ are the effective flexural rigidity of the biomorph part of the beam and the coupling coefficient term for parallel connections, respectively, and are given by

$$
\begin{aligned}
& E I_{c}=\frac{2 b}{3}\left\{E_{p} \frac{h_{p}^{3}}{8}+E_{1}\left[\left(h_{1}+\frac{h_{p}}{2}\right)^{3}-\frac{h_{p}^{3}}{8}\right]\right\} \\
& \vartheta=e_{31} b\left(h_{p}+h_{1}\right)
\end{aligned}
$$

where $e_{31}$ is the piezoelectric constant, $b$ is the width of both the piezoelectric layer and the beam, $h_{1}$ and $h_{p}$ are the thickness of the piezoelectric layer and the polymer beam, respectively, and $E_{1}$ is the modulus of elasticity of the piezoelectric layer.

\section{Magnetic Force}

The total magnetic force between two dipoles is a function of spatial derivatives of their magnetic field, whose formula is given in reference [80]. The total magnetic force is given by

$$
F_{m a g}=\frac{F_{R}}{Z^{4}}
$$

where $Z$ is the distance between the centers of the two magnets, $F_{R}$ is the magnitude of the moments for magnetic dipoles and given by

$$
F_{R}=\frac{3 \varepsilon m_{1} m_{2}}{2 \pi}
$$

where $m_{1}$ and $m_{2}$ are the moments of magnetic dipoles for the fixed and tip magnets, respectively. $\varepsilon$ is the permeability of the free space and equal to $4 \pi \times 10^{-7} \frac{\mathrm{mkg}}{\mathrm{s}^{2} \mathrm{~A}^{2}}$. As seen in Fig. 3, the total magnetic force can be written in terms of two components, one in the longitudinal direction, which is assumed to balance the longitudinal stiffness of the beam. The other one in the transverse direction, which is responsible for the beam transverse deflection and is given by Eqn. (6) 


$$
\begin{aligned}
F_{\text {magy }} & =F_{\text {mag }} \sin (\theta) \\
& =F_{\text {mag }} \frac{Y}{Z} \\
& =F_{m a g} \frac{Y}{\sqrt{\left(Y^{2}+d^{2}\right)}} \\
& =\frac{F_{R} Y}{\left(Y^{2}+d^{2}\right)^{5 / 2}}
\end{aligned}
$$

where $d$ is the longitudinal separation distance between the centers of the two magnets, and $Y$ is the deflection of the center of the moving magnet in the transverse direction given by

$$
Y=W_{2}(L, t)+L_{c} W_{2 x}(L, t)
$$

As it can be seen from Eqn. (6), the magnetic force is a nonlinear function of the beam tip displacement. This nonlinearity causes the system to have multiple equilibrium points.

\section{Static Analysis}

The static problem can be formulated by setting all time derivatives to zero in Eqn. (1) and Eqn. (2):

$$
\begin{aligned}
& E I_{c} W_{s 1 x x x x}(x)=0 \\
& E_{p} I_{p} W_{s 2 x x x x}(x)=0 \\
& W_{s 1}(0)=0, \quad W_{s 1 x}(0)=0 \\
& W_{s 1}\left(L_{1}\right)-W_{s 2}\left(L_{1}\right)=0 \\
& W_{s 1 x}\left(L_{1}\right)-W_{s 2 x}\left(L_{1}\right)=0 \\
& E I_{c} W_{s 1 x x}\left(L_{1}\right)-E_{p} I_{p} W_{s 2 x x}\left(L_{1}\right)=0 \\
& E I_{c} W_{s 1 x x x}\left(L_{1}\right)-E_{p} I_{p} W_{s 2 x x x}\left(L_{1}\right)=0 \\
& E_{p} I_{p} W_{s 2 x x x}(L)+F_{\text {magys }}-M g=0 \\
& E_{p} I_{p} W_{s 2 x x}(L)-L_{c} F_{\text {magys }}+L_{c} M g=0
\end{aligned}
$$

where the subscript $(s)$ refers to static, and $F_{\text {magys }}$ is the static form of the magnetic force in the transverse direction given by

$$
F_{\text {magys }}=\frac{F_{R} Y_{s}}{\left(Y_{s}^{2}+d^{2}\right)^{5 / 2}}
$$

where $Y_{S}$ is the static deflection for the magnetic tip center. Now the general solutions for the static equilibrium equations Eqn. (8) are third order polynomials of the forms:

$$
\begin{aligned}
& W_{s 1}(x)=A_{1} x^{3}+B_{1} x^{2}+C_{1} x+D_{1} \\
& W_{s 2}(x)=A_{2} x^{3}+B_{2} x^{2}+C_{2} x+D_{2}
\end{aligned}
$$

Applying the static boundary conditions in Eqn. (8), one can solve for all coefficients of $A_{1}, \ldots D_{2}$. The simulated static deflections were extracted from the solutions of Eqn. (10). The experimental static deflections of the tip magnet attached to 
Copyright (he beam with respect to the level of the beam base were measured when varying the distance between the two magnets. The threshold distance is specified as the farthest distance where the tip magnet can hold in both upper and lower potential wells.

Fig. 4 shows the variation of the static deflection for the center of the tip magnet with the distance between two magnets theoretically and experimentally. The maximum deflections obtained were $20 \mathrm{~mm}$ in both results. From the static response, it is clearly shown that there is a critical separation distance between the two magnets, called threshold separation distance $d_{t h}$, which has a value of $20 \mathrm{~mm}$. This threshold value divides the static response into two major regions. The first region $\left(d>d_{t h}\right)$ is called the mono-stable region, where the system oscillates around one stable equilibrium point in the middle. The second region $\left(d<d_{t h}\right)$ is called the bi-stable region, where the system oscillates between two stable equilibrium points. The static profile contains one stable branch for the mono-stable region and three branches for the bi-stable region; upper and lower branches are stable, while the middle one is unstable. The stability was examined by finding the eigenvalues of the Jacobian matrix at the equilibrium point and negative values indicated stability. The phenomenon of symmetry breaking bifurcation is shown clearly at the threshold value. The reason for this breaking is the weight of the system that acts as a constant force in the static equilibrium equation in addition to the nonlinearity caused by the magnetic force. If the weight were not present, the bifurcation diagram would be symmetric. Theoretical and experimental results are very close to each other and both results show the same threshold value $d_{t h}$, of $20 \mathrm{~mm}$.

\section{Eigenvalue Problem}

To determine the natural frequencies and mode shapes of the system, we solve the eigenvalue problem. The total deflection of the beam can be linearized around its equilibrium points as

$$
W(x, t)=W_{s}(x)+u(x, t)
$$

where $W_{s}(x)$ and $u(x, t)$ are the static equilibrium position and dynamic response, respectively. Substituting Eqn. (11) into Eqn. (7), we obtain the following form for the displacements for center of the moving magnet

$$
\begin{aligned}
Y & =W_{2 s}(L)+u_{2}(x, t)+L_{c} W_{2 s x}(L)+L_{c} u_{2 x}(x, t) \\
& =W_{2 s}(L)+L_{c} W_{2 s x}(L)+u_{2}(x, t)+L_{c} u_{2 x}(x, t) \\
& =Y_{s}+Y_{u}
\end{aligned}
$$

where $Y_{s}$ is the static displacement and $Y_{u}$ is the dynamic displacement of the magnet center. Substituting Eqn. 12 into Eqn. (6), and expanding in Taylor series around $Y_{u}=0$ and neglecting nonlinear terms yield

$$
\begin{aligned}
F_{\text {magy }} & =\frac{F_{R} Y s}{\left(d^{2}+Y_{s}^{2}\right)^{5 / 2}}+\frac{F_{R}\left(d^{2}-4 Y_{s}^{2}\right)}{\left(d^{2}+Y_{s}^{2}\right)^{7 / 2}} Y_{u} \\
& =F_{\text {magys }}+F_{\text {magyu }}
\end{aligned}
$$

where $F_{\text {magys }}$ and $F_{\text {magyu }}$ are the static and linearized dynamic magnetic forces, respectively. It should be noted that expansion of Eqn. (6) around zero will not yield the same result and will cause an error in the calculation of natural frequencies and dynamic responses. The key for correct expansion of the magnetic force is to account for the static equilibrium point in the equation. Eqn. (12) and (13) are then substituted into Eqn. (1) and Eqn. (2). Static magnetic force cancels the corresponding restoring force of the beam resulting in the following equations of motions and boundary conditions:

VIB-16-1454. Towfighian . 6 


$$
\begin{aligned}
& \rho A_{c} \ddot{u}_{1}(x, t)+E I_{c} u_{1 x x x x}(x, t)+c \dot{u}_{1}(x, t)-\vartheta V(t)\left[\frac{d \delta(x)}{d x}-\frac{d \delta(x-L)}{d x}\right]=0 \\
& \rho_{p} A_{p} \ddot{u}_{2}(x, t)+E_{p} I_{p} u_{2 x x x x}(x, t)+c \dot{u}_{2}(x, t)=0 \\
& C_{p} \dot{V}(t)+\left.\vartheta \frac{\partial \dot{u}_{1}(x, t)}{\partial x}\right|_{L_{1}}+\frac{V(t)}{R_{L}}=0 \\
& u_{1}(0, t)=0 \\
& u_{1 x}(0, t)=0 \\
& u_{1}\left(L_{1}, t\right)-u_{2}\left(L_{1}, t\right)=0 \\
& u_{1 x}\left(L_{1}, t\right)-u_{2 x}\left(L_{1}, t\right)=0 \\
& E I_{c} u_{1 x x}\left(L_{1}, t\right)-E_{p} I_{p} u_{2 x x}\left(L_{1}, t\right)=0 \\
& E I_{c} u_{1 x x x}\left(L_{1}, t\right)-E_{p} I_{p} u_{2 x x x}\left(L_{1}, t\right)=0 \\
& E_{p} I_{p} u_{2 x x x}(L, t)-M \ddot{u}_{2}(L, t)-M L_{c} \ddot{u}_{2 x}(L, t)+F_{\text {magyu }}=0 \\
& E_{p} I_{p} u_{2 x x}(L, t)+M L_{c} \ddot{u}_{2}(L, t)+\left[M L_{c}^{2}+J\right] \ddot{u}_{2 x}(L, t)-L_{c} F_{\text {magyu }}=0
\end{aligned}
$$
form of

Now to find the mode shapes and natural frequencies at a given static equilibrium position, we assume a solution in the

$$
u_{j}(x, t)=\phi_{j}(x) e^{i \omega t}
$$

where $\phi_{j}(x)$ is the first mode shape, and the subscript $j$ corresponds to the beam part. $\omega$ is the beam first natural frequency. Dropping the damping and voltage terms and substituting Eqn. (15) into Eqn. (14) we can extract the following eigenvalue problem

$$
\begin{aligned}
& \phi_{1 x x x x}(x)-a_{1}^{4} \phi_{1}(x)=0 \\
& \phi_{2 x x x x}(x)-a_{2}^{4} \phi_{2}(x)=0 \\
& \phi_{1}(0)=0 \\
& \phi_{1 x}(0)=0 \\
& \phi_{1}\left(L_{1}\right)-\phi_{2}\left(L_{1}\right)=0 \\
& \phi_{1 x}\left(L_{1}\right)-\phi_{2 x}\left(L_{1}\right)=0 \\
& E I_{c} \phi_{1 x x}\left(L_{1}\right)-E_{p} I_{p} \phi_{2 x x}\left(L_{1}\right)=0 \\
& E I_{c} \phi_{1 x x x}\left(L_{1}\right)-E_{p} I_{p} \phi_{2 x x x}\left(L_{1}\right)=0 \\
& \phi_{2 x x}(L)-L_{c} \eta \phi_{2}(L)-\frac{5}{3} L_{c}^{2} \eta \phi_{2 x}(L)-\frac{L_{c}}{E_{p} I_{p}} F_{\text {magy } \phi}=0 \\
& \phi_{2 x x x}(L)+\eta \phi_{2}(L)+L_{c} \eta \phi_{2 x}(L)+\frac{1}{E_{p} I_{p}} F_{\text {magy } \phi}=0
\end{aligned}
$$

where

$$
a_{1}^{4}=\frac{\rho A_{c} \omega^{2}}{E I_{c}}, \quad a_{2}^{4}=\frac{\rho_{p} A_{p} \omega^{2}}{E_{p} I_{p}}
$$

and $\eta=\frac{M \omega^{2}}{E_{p} I_{p}}, F_{\text {magy } \phi}$ is the magnetic force as a function of the mode shape and is given by

$$
F_{\text {magy } \phi}=\frac{F_{R}\left(d^{2}-4 Y_{s}^{2}\right)}{\left(d^{2}+Y_{s}^{2}\right)^{7 / 2}} Y_{\phi}
$$




$$
Y_{\phi}=\phi(L)+L_{c} \phi_{x}(L)
$$

Fig. 5 shows the first normalized mode shape plotted at some sample values in the mono-stable, bi-stable, and transition regions to compare the mode shape magnitudes for these three regimes. The mode shapes are normalized with respect to the maximum mode amplitude among all distances. The higher amplitude and bending effect shown in the bi-stable region $(d=6 \mathrm{~mm})$ are due to the higher magnetic force value at this region. The smaller the distance, the larger the amplitude in the bi-stable region, which is consistent with the results in Fig. 4. The smallest amplitude among all distances was at the threshold distance $(d=20 \mathrm{~mm})$. The first mode shape profile is consistent with the experimentally observed oscillations and hence will be used as the dominant mode shape for the dynamic analysis. The natural frequency for the first mode shape of the linear system (when the fixed magnet is removed, but the moving magnet and its weight effect is present) was calculated by solving the system of Eqn. (16) after equating the magnetic force term to zero. It was found that the linear resonator has a natural frequency of $17.35 \mathrm{~Hz}$.

Next, we investigated the variation of the natural frequency of the nonlinear resonator under the effect of the magnetic force that varies by the distance between the two magnets. Different researchers studied the frequency variation with changing the separation distance between the two magnets [34,45,72]. However, these studies didn't provide a match between the experimental and simulated results. Fig. 6 shows the theoretical and experimental variation of the first natural frequency with the distance between the two magnets. The results extracted theoretically from the solving the eigenvalue problem and experimentally with an excitation amplitude of $0.5 \mathrm{~g}$ are in very good agreement. The results show a threshold separation distance, $d_{t h}, 20 \mathrm{~mm}$, which separates the bi-stable and mono-stable regions. The magnetic force has its maximum effect on the system in the bi-stable region at smaller distances. At this point the natural frequency starts with a higher value of $38 \mathrm{~Hz}$ and starts to drop by increasing the distance until it reaches its minimum value of $15 \mathrm{~Hz}$ at the threshold distance experimentally. Increasing the distance further will move the system to the mono-stable region, where the natural frequency starts to increase and converges to that of the linear resonator where the magnetic force has no effect.

\section{Dynamic Analysis}

\subsection{Reduced Order Model (ROM)}

To evaluate the dynamic response of the resonator and the produced voltage by the piezoelectric layer, the governing equations for the forced vibration are derived using Lagrange's equations. The total kinetic and potential energy for the harvester is divided into two parts, the first for the biomorph beam and the second for the polymer beam. At the end, the two governing equations are merged into one governing equation in addition to the electrical equation of the piezoelectric layers. For the first part of the beam (biomorph portion), the kinetic and potential energy are given by $T_{1}$ and $U_{1}$, respectively:

$$
\begin{gathered}
T_{1}=\int_{0}^{L_{1}} \frac{1}{2} \rho A_{c} \dot{W}_{1}^{2}(x, t) d x \\
U_{1}=\int_{0}^{L_{1}} \frac{1}{2} E I_{c} W_{1 x x}^{2}(x, t) d x-\vartheta V(t) W_{1 x}\left(L_{1}, t\right)-\frac{1}{2} C_{p} V^{2}(t)
\end{gathered}
$$

where the last two terms in Eqn. (21) are the potential energy for the piezoelectric layers. For the second part of the beam (polymer layer), $T_{2}$ and $U_{2}$ are given by:

$$
\begin{gathered}
T_{2}=\int_{L_{1}}^{L} \frac{1}{2} \rho_{p} A_{P} \dot{W}_{2}^{2}(x, t) d x+\frac{1}{2} M\left[\dot{W}_{2}(L, t)+L_{c} \dot{W}_{2 x}(L, t)\right]^{2}+\frac{1}{2} J \dot{W}_{2 x}^{2}(L, t) \\
U_{2}=\int_{L_{1}}^{L} \frac{1}{2} E_{p} I_{p} W_{2 x x}^{2}(x, t) d x+U_{M a g}
\end{gathered}
$$

VIB-16-1454 . Towfighian . 8 
Copyright (chere, $U_{\text {Mag }}$ is the magnetic potential energy. The total kinetic energy is given by $T=T_{1}+T_{2}$, while the total potential energy is given by $U=U_{1}+U_{2}$. Lagrange's equation is written as $\ell=T-U$. To develop a ROM for the energy harvester, we need to express the transverse deflection using the Galerkin approach as

$$
\begin{array}{ll}
W_{1}(x, t)=W_{1 s}(x)+\sum_{i=1}^{n} \phi_{1 i}(x) q_{i}(t)+Z(t), & 0 \leq x \leq L_{1} \\
W_{2}(x, t)=W_{2 s}(x)+\sum_{i=1}^{n} \phi_{2 i}(x) q_{i}(t)+Z(t), & L_{1} \leq x \leq L
\end{array}
$$

where $q_{i}(t)$ is the modal coordinate, $Z(t)$ is the base motion, and $n$ is the number of mode shapes. Because of the mass of the moving magnet, the beam's first mode will be dominant and has the greatest strain profile near the clamped end, which means largest piezoelectric response (higher electrical energy). Because of the dominance of the first mode, the governing equations will be approximated to one mode shape. Using Lagrange's equation with one mode approximation, we can obtain

$$
\begin{aligned}
& \ddot{q}_{1}(t)+2 \mu \omega_{n} \dot{q}_{1}(t)+\omega_{n}^{2} q_{1}(t)-\chi V(t)-F_{0} \cos (\Omega t)+\frac{1}{M_{33}} F_{\text {magyu }}=0 \\
& \dot{V}(t)+\kappa \dot{q}_{1}(t)+\lambda V(t)=0
\end{aligned}
$$

where

$$
\begin{gathered}
\mu=\frac{D_{33}}{2 M_{33} \omega_{n}}, \quad \omega_{n}=\sqrt{\frac{K_{33}+\alpha_{1}}{M_{33}}}, \quad \chi=\frac{\theta_{33}}{M_{33}}, \\
\alpha_{1}=\frac{F_{R}\left(d^{2}-4 Y_{s}^{2}\right)}{\left(d^{2}+Y_{s}^{2}\right)^{7 / 2}} Y_{\phi}, \quad F_{0}=\frac{A \Omega^{2} H_{33}}{M_{33}}, \quad \lambda=\frac{1}{C_{p} R_{L}}, \quad \kappa=\frac{\theta_{33}}{C_{p}}
\end{gathered}
$$

where $\alpha_{1}$ is the linear part of the magnetic force after Taylor series expansion. This term shows how the natural frequency is affected by the distance between magnets. The remaining coefficients of Eqn. (25) are given by

$$
\begin{gathered}
M_{33}=\int_{0}^{L_{1}} \rho A_{c} \phi_{1}^{2}(x) d x+\int_{L_{1}}^{L} \rho_{p} A_{p} \phi_{2}^{2}(x) d x+M\left(\phi_{2}(L)+L_{c} \phi_{2 x}(L)\right)^{2}+J \phi_{2 x}^{2}(L) \\
D_{33}=c \int_{0}^{L_{1}} \phi_{1}^{2}(x) d x+c \int_{L_{1}}^{L} \phi_{2}^{2}(x) d x \\
K_{33}=\int_{0}^{L_{1}} E I_{c} \phi_{1 x x}^{2}(x) d x+\int_{L_{1}}^{L} E_{p} I_{p} \phi_{2 x x}^{2}(x) d x \\
\theta_{33}=\vartheta \phi_{1 x}\left(L_{1}\right) \\
H_{33}=\int_{0}^{L_{1}} \rho A_{c} \phi_{1}(x) d x+M\left(\phi_{2}(L)+L_{c} \phi_{2 x}(L)\right)+\int_{L_{1}}^{L} \rho_{p} A_{p} \phi_{2}(x) d x \\
F_{\text {magyu }}=\alpha_{2} q_{1}^{2}(t)+\alpha_{3} q_{1}^{3}(t)+\alpha_{4} q_{1}^{4}(t)+\alpha_{5} q_{1}^{5}(t)+\alpha_{6} q_{1}^{6}(t)+\alpha_{7} q_{1}^{7}(t)+ \\
\alpha_{8} q_{1}^{8}(t)+\alpha_{9} q_{1}^{9}(t)+O\left(q_{1}^{10}\right)
\end{gathered}
$$

where $\alpha_{1}, \ldots, \alpha_{9}$ are the coefficients of Taylor's series expansion, listed in the Appendix. It's worth mentioning that the quadratic and cubic terms dominate and have the largest effect on the dynamics of the harvester. 
Copyright (Difrerent techniques can be used to solve the system of equations such as Long Time Integration (LTI) and the Method of Harmonic Balance (MHB) $[57,73,74]$. However, such techniques have some limitations. In addition to more computational time, LTI cannot catch the unstable solution. MHB depends on the number of terms in the assumed harmonic solution. To catch the nonlinear response with good accuracy, a higher number of harmonics would have to be taken into consideration, which in turn will make the problem very sophisticated and difficult to solve. Because the nonlinear behavior of our harvester is essential to increase the bandwidth and amplitude, which leads to better designs, it is important to closely capture these nonlinear behaviors. For such a goal, we use the shooting technique for obtaining stable and unstable solutions and analyzing their stability for the nonlinear resonator. Also, to guarantee the accuracy of the shooting results, it will be compared to the long time integration method results. To apply the shooting method, the governing equations, Eqn. (25), should be written in state space form, which lead to the following three first order ordinary differential equations (ODEs):

$$
\begin{aligned}
& \dot{X}_{1}=X_{2} \\
& \dot{X}_{2}=-\left(2 \mu \omega_{n} X_{2}+\omega_{n}^{2} X_{1}-\chi X_{3}+F_{0} \cos (\Omega t)+\frac{1}{M_{33}} F_{\text {maguX }}\right) \\
& \dot{X}_{3}=-\left(\kappa X_{2}+\lambda X_{3}\right)
\end{aligned}
$$

where $F_{\text {maguX }}$ is the magnetic force but written in state space form up to the ninth order term and given by

$$
\begin{aligned}
F_{\text {magyX }}= & \alpha_{2} X_{1}^{2}(t)+\alpha_{3} X_{1}^{3}(t)+\alpha_{4} X_{1}^{4}(t)+\alpha_{5} X_{1}^{5}(t)+ \\
& \alpha_{6} X_{1}^{6}(t)+\alpha_{7} X_{1}^{7}(t)+\alpha_{8} X_{1}^{8}(t)+\alpha_{9} X_{1}^{9}(t)
\end{aligned}
$$

Then, by following the procedures described in [81] we end up with a first order ODE system of equations with their related initial conditions. These equations are integrated numerically using an initial guess for the initial conditions over one period $T$. Where $T=\frac{2 \pi}{\Omega}$, and $\Omega$ is the excitation frequency. The process is repeated until convergence is achieved and the errors are minimized. More precisely, a converged solution is reached when the error becomes less than a preset tolerance. An ordinary differential equation solver in the software Mathematica [82] based on the Runge-Kutta scheme is used to integrate the dynamical system. The next step is analyzing the stability for the obtained periodic solution. This is done by solving for the eigenvalues, called Floquet multipliers, of the Manodromy matrix. Three Floquet multipliers will be the output of the eigenvalues for the Manodromy matrix, and the solution is considered stable if the absolute value for all Floquet multipliers are less than one, otherwise the solution is considered unstable.

\section{Results and Discussions}

\subsection{Linear Harvester}

Using the experimental setup in Fig. 2, the resonance frequency was found to be $17.45 \mathrm{~Hz}$ at the maximum peak-to-peak deflection of $9.5 \mathrm{~mm}$ with a maximum peak-to-peak voltage of $2.4 \mathrm{~V}$ at $0.5 \mathrm{~g}$ base excitation.

It should be noted that the beam entirely made of piezoelectric laminate with the same length has a natural frequency of around $100 \mathrm{~Hz}$ compared to $17.45 \mathrm{~Hz}$ for the proposed beam with extended polymer. That means that our harvester lowered the natural frequency by $87 \%$, making that more suitable for low ambient and wildlife frequencies such as those of bird flights for self-powered tag applications. This natural frequency can be decreased further as we decrease the distance between two magnets close to the threshold distance, where a wide bandwidth can be realized as explained next.

\subsection{Nonlinear Harvester}

Next, we investigate the dynamics of the energy harvester for different distances in the mono-stable and bi-stable regions. We picked two values for $d$ in the mono-stable and the bi-stable regions as shown in Fig. 4. The frequency response and open circuit voltage response were investigated for each distance at different excitation levels.

\subsubsection{Mono-Stable Cases}

The dynamics of the energy harvester in the mono-stable region is explored for a separation distance of $40 \mathrm{~mm}$ between the two magnets. Fig. 7a and Fig. 7b show the forward swept experimental and simulated displacement and the voltage responses, respectively, under $0.5 \mathrm{~g}$ excitation level. Simulation results were conducted with shooting method. Simulated 

amplitude, but the voltage output is smaller compared to the linear resonator for the same excitation level of $0.5 \mathrm{~g}$. This can be explained by referring to the third equation in Equation set of (14). In this ODE equation, the output voltage varies with the tip angular velocity term (frequency). One can deduce that although the displacement amplitude has increased in the mono-stable region compared to a linear resonator, because of the frequency decrease, the voltage has decreased. The predicted output voltage in Fig. 7b seems to be less accurate compared to the experimental results, and the reason for this is that in the model the piezoelectric layer is assumed clamped, which in the experiment, it is very close to the base, but not clamped with other beam layers.

Fig. 8a and Fig. 8b show the experimental displacement and voltage responses under different excitation levels. Higher amplitude is achieved by increasing the excitation level. Hardening phenomenon is noticed when increasing the excitation level in both responses.

Next, the distance between the two magnets will be reduced toward the threshold from the mono-stable side to $d=22 \mathrm{~mm}$, Fig. 9. As seen, despite the lower deflection amplitude, the voltage amplitude has increased to $5.4 \mathrm{~V}$. The reason is that the voltage is a function of angular velocity at the end of piezoelectric equation Eqn. (1) and not the tip displacement. Hence, although the amplitude dropped, the voltage increase occurs because of increase in the angular velocity.

\subsubsection{Bi-Stable Case}

In this section, we will investigate the dynamic behavior of the energy harvester in the bi-stable region. We will start by analyzing the dynamics of the smallest distance between the two magnets then we increase the separation toward the threshold value in the next subsection. The frequency and voltage curves shown in Fig. 10a and Fig. 10b, respectively, are for $d=5 \mathrm{~mm}$. One can notice the higher shift in the natural frequency value to $34 \mathrm{~Hz}$ for the nonlinear harvester compared to $17 \mathrm{~Hz}$ for the linear resonator. The amplitude of the deflection is very low compared to the mono-stable results. This can be explained from the oscillations being confined around the bottom equilibrium point because of the effect of the weight and larger magnetic force at small distances. Softening behavior can be seen clearly in the two curves. At very low separation distances between the two magnets, the magnetic force expression does not obey the formula used in the equation and it becomes very complicated. Because of this, no periodic solution can be obtained by the model.

\subsubsection{Transition Region}

We then examine the response in the bi-stable regime at a separation distance very close to the threshold, $d=18 \mathrm{~mm}$, (transition region). Experimental and simulated results at $0.5 \mathrm{~g}$ excitation level are shown for the displacement and voltage responses in Fig. 11a and Fig. 11b, respectively. Good qualitative agreement is shown between the experimental and simulated results showing the softening behavior. Less accurate prediction of the voltage could be because in the experiment the piezoelectric strip is very close to the base of the beam, but it is not clamped in the experiment. We avoided clamping the piezoelectric strip as that led to breakage at high excitation levels. In theory, all layers of the beam consisting of polymer and the piezoelectric strip are perfectly clamped at the base. This difference in the boundary results in smaller voltage in practice.

At the transition distance in the bi-stable region $(d=18 \mathrm{~mm})$, the nature of the frequency response at higher excitation level, such as $1.0 \mathrm{~g}$, changes from softening to hardening as shown in Fig. 12a and Fig. 12b. Using the shooting technique, we captured the stable and unstable solutions. The figures reveal the nonuniqueness of the solution for the nonlinear harvester. In some frequency ranges, three solutions are available, two of which are stable (black circle), and one is unstable (red square). Sweeping the frequency in a forward direction from point $(a)$ and a backward direction from point $(d)$ will result in the $b-c-e-f$ hysteresis loop as shown in Fig. 12a and Fig. 12b. The nonlinearity caused by the magnetic force increases the harvester's bandwidth compared with the harvester bandwidth in Fig. 7a and Fig. 7b.

In Fig. 12, we notice the hysteresis and multiple solutions behavior because of the nonlinearity in the system. For such regions with multiple solutions, the periodic solution amplitude depends on the initial conditions. As an example, in Fig. 12a at a frequency of $18 \mathrm{~Hz}$ the system can converge to the lower branch or upper branch depending on the initial conditions. For such cases the basin of attraction is considered as a powerful tool to examine the multiple solutions convergence based on varying the initial conditions. Fig. 13a shows the basin of attraction for the two solutions at $\Omega=18 \mathrm{~Hz}$, where the orange color represents the lower branch convergence and the black color is the upper branch convergence obtained for long time integration. The figure shows the basin of attraction of the upper branch is slightly smaller than that of the lower branch; however, it has a comparable size. Decreasing the frequency to the fold frequency at $16.7 \mathrm{~Hz}$, the basin of attraction of the upper branch becomes much larger than the lower branch (Fig. 13b). 
The experimental, simulated results from the shooting method, and simulated results from long time integration for the transition separation distance of $18 \mathrm{~mm}$ at different excitation levels are shown in Figures 14, 15 and 16, respectively. The results from shooting method and numerical integration are in close agreement. The shooting method is superior because of its capability of catching the unstable branch and its low computational time. In the transition region, the harvester gets higher deflection than that of the two previous cases in the bi-stable region. Maximum amplitude and voltage achieved were $34 \mathrm{~mm}$ and $7.0 \mathrm{~V}$, respectively at excitation level of $1.0 \mathrm{~g}$. We observe a combined nonlinear behavior of softening and hardening as a function of the excitation level. At low excitation levels, the quadratic nonlinearity dominates, so softening occurs, while at high excitation levels, positive cubic nonlinearity dominates and hardening appears. This observation was made possible by considering the quadratic nonlinearity of the magnetic force, which has not reported in previous studies. [47-59].

Higher bandwidth is also obtained at the transition region, especially at high excitation levels. At medium excitation levels, such as $0.5 \mathrm{~g}$, a $35 \%$ increase in the bandwidth and a $37 \%$ increase in the maximum amplitude are attained compared to the linear resonator. Qualitatively, the experimental results in Figures (14a, 14b) are in good agreement with theoretical results in Figures $(15 \mathrm{a}, 15 \mathrm{~b})$. However, quantitatively these results are not close to each other. The reasons for such different can be explained because of the deflection measurements. The center of the tip magnet theoretically is assumed to be aligned with the neutral axis of the cantilever beam, while experimentally it is placed on top of the free end of the cantilever beam. Another reason for the difference is the boundary conditions in the theoretical model, which are assumed to be fully clamped-free case, while in experiment such boundary is not ideally clamped.

To assess the role of resistance on the harvester output, we vary the resistance from the $1 M \Omega$ used for our simulations so far. Increasing the resistance will increase the amount of voltage produced as shown in Figure 17. We can notice that the output voltage duplicates by increasing the resistance from $1 M \Omega$ to $10 M \Omega$. A further increase will not yield a significant difference in the generated voltage, thus the optimum resistance value is found to be $10 \mathrm{M} \Omega$.

The behavior of the system at the threshold distance $\left(d_{t h}=20 \mathrm{~mm}\right)$ has distinct features. This is the distance where the twowell-potential transitions to the one-well-potential function. Small changes in some parameters lead to period doubling and chaos. We use phase portraits, Poincaré sections, and bifurcation diagrams to characterize the dynamic responses. Keeping the frequency constant at the natural frequency at the threshold distance (Fig. 6), $12.59 \mathrm{~Hz}$, we vary the level of excitation to obtain the force sweep, see Fig 18. This figure is obtained from long time integration for 650 periods of excitation using the initial conditions for periodic solutions of the shooting technique and then recording the last 130 periods at the steady-sate region. Poincaré sections are taken by sampling the data at the period of excitation starting from the displacement with the maximum absolute magnitude. The nature of the response depends on the initial conditions, the magnitude of the excitation, and the frequency of excitation. The excitation level starts at $0.2 \mathrm{~g}$ where the harvester oscillates in one potential well at the frequency of excitation. By increasing the level of excitation, the periodic solution evolves into period doubling.

The increase of excitation level beyond the period doubling cascade (period 2, 4 and 8) leads to the appearance of chaotic oscillation as seen in Fig 18. As it is deduced, the chaotic response has two bands, a lower band around the lower stable equilibrium point and the upper one around the saddle. The size of the upper band is larger than the lower band. This fact is also seen from the number of crossings of the zero-velocity axis around the $-0.7 \mathrm{~mm}$ and $-1.4 \mathrm{~mm}$ in Fig. 19aa. The phase portrait shows irregular excursions of the chaotic response, while the Poincare section in Fig. 19b clearly reveals the chaotic attractor.

Similar period doubling and chaotic behaviors are observed by sweeping the frequency of excitation at the fixed excitation level of $0.2 \mathrm{~g}$, see Fig. 20 at the threshold distance, $d_{t h}=20 \mathrm{~mm}$. The frequency is decreased toward the natural frequency of the threshold distance. Results indicate that the system has a period doubling bifurcation at the natural frequency, $12.51 \mathrm{~Hz}$. Consequently, period 2, period 4 and period 8 are visible in the period doubling cascade that culminates in chaos as the frequency decreases further. The chaotic response is an unpredictable response and very sensitive to the initial conditions.

In summary, we can conclude that the higher energy can be harvested in the mono-stable and bi-stable regions at distances close to the threshold value. In other words, the optimum range for harvesting energy was found to be around the threshold distance from both sides, mono and bi-stable regions. To illustrate this result, the maximum output voltages at the excitation level of $1.0 \mathrm{~g}$ were recorded for all previous cases in the mono and bi-stable regions (Fig. 21a). Results indicate the maximum voltage of $7 \mathrm{~V}$ can be obtained in the bi-stable region close to the threshold distance of $20 \mathrm{~mm}$.

Another interesting conclusion is related to the dynamic behavior of the energy harvester. It was noticed that the hardening behavior is the dominant case for the mono-stable region (at large distances), while the softening behavior is the prominent behavior in the bi-stable region (at small distances). As the distances get closer to the threshold from both sides (the mono and bi-stable regions) a combination of hardening and softening behaviors appears with a dominant hardening response. Results are summarized in Fig. 21b. Tuning the frequency to the transition region enables controlling the natural frequency

VIB-16-1454. Towfighian . 12 
Copyright (fo the harvester to match the low frequency range of interest for different applications. The increased nonlinearities in this region (combination behavior) leads to an increase in the frequency bandwidth and the amount of energy harvested.

\section{Conclusions}

A magnetoelastic piezoelectric energy harvester was investigated in this paper to increase the frequency bandwidth and amplitude of the response. A mathematical model for the continuous system of the energy harvester was developed. Static analysis of the harvester revealed a threshold value that divided the nature of the response into mono- and bi-stable regimes based on the distance between two magnets. Thorough attention was paid to the magnetic force and its interactions with the beam dynamics, and that led us to accurately predict the variation of the fundamental natural frequency with the distance between two magnets. Consequently, frequency response simulations of the system were obtained with high accuracy using the shooting technique. The shooting method enabled us to capture stable and unstable solutions and obtain a good agreement with experimental results on softening, hardening and combination behavior across bi-stable, mono-stable and transition regimes, respectively. For the first time, we report on the combination of softening and hardening behavior in the transition region (where mono-stability changes to bi-stability). Because the output voltage and frequency bandwidth are the largest near the transition region, this region was found to be the optimum range for harvesting energy. Compared to a linear resonator at medium excitation level of $0.5 \mathrm{~g}$, an increment of $37 \%$ in the output voltage and an increase of $35 \%$ in the frequency bandwidth was obtained at distances close to the threshold distance. In addition, the natural frequency of the resonator energy harvester was decreased from $100 \mathrm{~Hz}$ to $17 \mathrm{~Hz}$ by operating at the transition region, which makes it more suitable for energy harvesting from ambient vibrations.

\section{References}

[1] Shafer, M. W., MacCurdy, R., Shipley, J. R., Winkler, D., Guglielmo, C. G., and Garcia, E., 2015. "The case for energy harvesting on wildlife in flight". Smart Materials and Structures, 24(2), p. 025031.

[2] Roundy, S., Wright, P. K., and Rabaey, J., 2003. "A study of low level vibrations as a power source for wireless sensor nodes". Computer communications, 26(11), pp. 1131-1144.

[3] Mitcheson, P. D., Green, T. C., Yeatman, E. M., and Holmes, A. S., 2004. "Architectures for vibration-driven micropower generators". Microelectromechanical Systems, Journal of, 13(3), pp. 429-440.

[4] Cook-Chennault, K., Thambi, N., and Sastry, A., 2008. "Powering mems portable devicesa review of non-regenerative and regenerative power supply systems with special emphasis on piezoelectric energy harvesting systems". Smart Materials and Structures, 17(4), p. 043001.

[5] González, J. L., Rubio, A., and Moll, F., 2002. "Human powered piezoelectric batteries to supply power to wearable electronic devices". International journal of the Society of Materials Engineering for Resources, 10(1), pp. 34-40.

[6] Mathúna, C. Ó., ODonnell, T., Martinez-Catala, R. V., Rohan, J., and OFlynn, B., 2008. "Energy scavenging for long-term deployable wireless sensor networks". Talanta, 75(3), pp. 613-623.

[7] Torah, R., Glynne-Jones, P., Tudor, M., O’Donnell, T., Roy, S., and Beeby, S., 2008. "Self-powered autonomous wireless sensor node using vibration energy harvesting”. Measurement science and technology, 19(12), p. 125202.

[8] Gregori, S., Li, Y., Li, H., Liu, J., and Maloberti, F., 2004. "2.45 ghz power and data transmission for a low-power autonomous sensors platform”. In Low Power Electronics and Design, 2004. ISLPED’04. Proceedings of the 2004 International Symposium on, IEEE, pp. 269-273.

[9] Kim, J.-W., Takao, H., Sawada, K., and Ishida, M., 2007. "Integrated inductors for rf transmitters in cmos/mems smart microsensor systems". Sensors, 7(8), pp. 1387-1398.

[10] Bracke, W., Merken, P., Puers, R., and Van Hoof, C., 2007. "Generic architectures and design methods for autonomous sensors". Sensors and Actuators A: Physical, 135(2), pp. 881-888.

[11] Baert, K., Gyselinckx, B., Torfs, T., Leonov, V., Yazicioglu, F., Brebels, S., Donnay, S., Vanfleteren, J., Beyne, E., and Van Hoof, C., 2006. "Technologies for highly miniaturized autonomous sensor networks". Microelectronics Journal, 37(12), pp. 1563-1568.

[12] Sodano, H. A., Inman, D. J., and Park, G., 2004. "A review of power harvesting from vibration using piezoelectric materials". Shock and Vibration Digest, 36(3), pp. 197-206.

[13] Sodano, H. A., Inman, D. J., and Park, G., 2005. "Generation and storage of electricity from power harvesting devices". Journal of Intelligent Material Systems and Structures, 16(1), pp. 67-75.

[14] Roundy, S., 2005. "On the effectiveness of vibration-based energy harvesting”. Journal of intelligent material systems and structures, 16(10), pp. 809-823.

[15] Daqaq, M. F., 2010. "Response of uni-modal duffing-type harvesters to random forced excitations". Journal of Sound and Vibration, 329(18), pp. 3621-3631.

[16] Mann, B., and Owens, B., 2010. "Investigations of a nonlinear energy harvester with a bistable potential well". Journal of Sound and Vibration, 329(9), pp. 1215-1226. 
Copyright (17] Cammarano, A., Burrow, S., and Barton, D., 2011. "Modelling and experimental characterization of an energy harvester with bi-stable compliance characteristics". Proceedings of the Institution of Mechanical Engineers, Part I: Journal of Systems and Control Engineering, 225(4), pp. 475-484.

[18] Ando, B., Baglio, S., Trigona, C., Dumas, N., Latorre, L., and Nouet, P., 2010. "Nonlinear mechanism in mems devices for energy harvesting applications". Journal of Micromechanics and Microengineering, 20(12), p. 125020.

[19] Nguyen, S. D., Halvorsen, E., and Paprotny, I., 2013. "Bistable springs for wideband microelectromechanical energy harvesters". Applied Physics Letters, 102(2), p. 023904.

[20] Ibrahim, A., Towfighian, S., Younis, M., and Su, Q., 2016. "Magnetoelastic beam with extended polymer for low frequency vibration energy harvesting”. In SPIE Smart Structures and Materials+ Nondestructive Evaluation and Health Monitoring, International Society for Optics and Photonics, pp. 98060B-98060B.

[21] Beeby, S. P., Tudor, M. J., and White, N., 2006. "Energy harvesting vibration sources for microsystems applications". Measurement science and technology, 17(12), p. R175.

[22] Mitcheson, P. D., Yeatman, E. M., Rao, G. K., Holmes, A. S., and Green, T. C., 2008. "Energy harvesting from human and machine motion for wireless electronic devices”. Proceedings of the IEEE, 96(9), pp. 1457-1486.

[23] Sterken, T., Baert, K., Van Hoof, C., Puers, R., Borghs, G., and Fiorini, P., 2004. "Comparative modelling for vibration scavengers [mems energy scavengers]". In Sensors, 2004. Proceedings of IEEE, IEEE, pp. 1249-1252.

[24] James, E., Tudor, M., Beeby, S., Harris, N., Glynne-Jones, P., Ross, J., and White, N., 2004. "An investigation of self-powered systems for condition monitoring applications". Sensors and Actuators A: Physical, 110(1), pp. 171-176.

[25] Roundy, S., and Wright, P. K., 2004. "A piezoelectric vibration based generator for wireless electronics". Smart Materials and structures, 13(5), p. 1131.

[26] Ottman, G. K., Hofmann, H. F., Bhatt, A. C., and Lesieutre, G. A., 2002. "Adaptive piezoelectric energy harvesting circuit for wireless remote power supply". Power Electronics, IEEE Transactions on, 17(5), pp. 669-676.

[27] Ferrari, M., Ferrari, V., Marioli, D., and Taroni, A., 2006. "Modeling, fabrication and performance measurements of a piezoelectric energy converter for power harvesting in autonomous microsystems". IEEE transactions on instrumentation and measurement, 55(6), pp. 2096-2101.

[28] Ferrari, M., Ferrari, V., Guizzetti, M., and Marioli, D., 2009. "An autonomous battery-less sensor module powered by piezoelectric energy harvesting with rf transmission of multiple measurement signals". Smart Materials and Structures, 18(8), p. 085023.

[29] Masana, R., and Daqaq, M. F., 2011. "Relative performance of a vibratory energy harvester in mono-and bi-stable potentials". Journal of Sound and Vibration, 330(24), pp. 6036-6052.

[30] Sneller, A., Cette, P., and Mann, B., 2011. "Experimental investigation of a post-buckled piezoelectric beam with an attached central mass used to harvest energy". Proceedings of the Institution of Mechanical Engineers, Part I: Journal of Systems and Control Engineering, 225(4), pp. 497-509.

[31] Arrieta, A., Hagedorn, P., Erturk, A., and Inman, D., 2010. "A piezoelectric bistable plate for nonlinear broadband energy harvesting”. Applied Physics Letters, 97(10), p. 104102.

[32] Masana, R., and Daqaq, M. F., 2012. "Energy harvesting in the super-harmonic frequency region of a twin-well oscillator". Journal of Applied Physics, 111(4), p. 044501.

[33] Ferrari, M., Bau, M., Guizzetti, M., and Ferrari, V., 2011. "A single-magnet nonlinear piezoelectric converter for enhanced energy harvesting from random vibrations”. Sensors and Actuators A: Physical, 172(1), pp. $287-292$.

[34] Ferrari, M., Ferrari, V., Guizzetti, M., Andò, B., Baglio, S., and Trigona, C., 2010. "Improved energy harvesting from wideband vibrations by nonlinear piezoelectric converters". Sensors and Actuators A: Physical, 162(2), pp. $425-431$.

[35] Litak, G., Friswell, M., and Adhikari, S., 2010. "Magnetopiezoelastic energy harvesting driven by random excitations". Applied Physics Letters, 96(21), p. 214103.

[36] Lin, J.-T., Lee, B., and Alphenaar, B., 2010. "The magnetic coupling of a piezoelectric cantilever for enhanced energy harvesting efficiency". Smart materials and Structures, 19(4), p. 045012.

[37] Ali, S., Adhikari, S., Friswell, M., and Narayanan, S., 2011. "The analysis of piezomagnetoelastic energy harvesters under broadband random excitations". Journal of Applied Physics, 109(7), p. 074904.

[38] Roundy, S., and Zhang, Y., 2005. "Toward self-tuning adaptive vibration-based microgenerators". In Smart Materials, Nano-, and Micro-Smart Systems, International Society for Optics and Photonics, pp. 373-384.

[39] Wu, W.-J., Chen, Y.-Y., Lee, B.-S., He, J.-J., and Peng, Y.-T., 2006. "Tunable resonant frequency power harvesting devices". In Smart Structures and Materials, International Society for Optics and Photonics, pp. 61690A-61690A.

[40] Challa, V. R., Prasad, M., Shi, Y., and Fisher, F. T., 2008. "A vibration energy harvesting device with bidirectional resonance frequency tunability". Smart Materials and Structures, 17(1), p. 015035.

[41] Shahruz, S., 2006. "Design of mechanical band-pass filters for energy scavenging". Journal of Sound and Vibration, 292(3), pp. 987-998.

[42] Shahruz, S., 2006. "Limits of performance of mechanical band-pass filters used in energy scavenging". Journal of sound and vibration, 293(1), pp. 449-461.

[43] Baker, J., Roundy, S., Wright, P., et al., 2005. "Alternative geometries for increasing power density in vibration energy

VIB-16-1454 . Towfighian . 14 

Aug.), pp. 959-70.

[44] Rastegar, J., Pereira, C., and Nguyen, H.-L., 2006. "Piezoelectric-based power sources for harvesting energy from platforms with low-frequency vibration”. In Smart Structures and Materials, International Society for Optics and Photonics, pp. 617101-617101.

[45] Al-Ashtari, W., Hunstig, M., Hemsel, T., and Sextro, W., 2012. "Frequency tuning of piezoelectric energy harvesters by magnetic force". Smart Materials and Structures, 21(3), p. 035019.

[46] Yang, W., and Towfighian, S., 2017. “A hybrid nonlinear vibration energy harvester”. Mechanical Systems and Signal Processing, 90, pp. 317-333.

[47] Moon, F., and Holmes, P. J., 1979. “A magnetoelastic strange attractor”. Journal of Sound and Vibration, 65(2), pp. 275-296.

[48] Quinn, D. D., Vakakis, A. F., and Bergman, L. A., 2007. "Vibration-based energy harvesting with essential nonlinearities". In ASME 2007 International Design Engineering Technical Conferences and Computers and Information in Engineering Conference, American Society of Mechanical Engineers, pp. 779-786.

[49] Mann, B., and Sims, N., 2009. "Energy harvesting from the nonlinear oscillations of magnetic levitation". Journal of Sound and Vibration, 319(1), pp. 515-530.

[50] Burrow, S., and Clare, L., 2007. "A resonant generator with non-linear compliance for energy harvesting in high vibrational environments". In 2007 IEEE International Electric Machines \& Drives Conference, Vol. 1, IEEE, pp. 715720.

[51] Beeby, S. P., Torah, R., Tudor, M., Glynne-Jones, P., O’Donnell, T., Saha, C., and Roy, S., 2007. “A micro electromagnetic generator for vibration energy harvesting". Journal of Micromechanics and microengineering, 17(7), p. 1257.

[52] Cottone, F., Vocca, H., and Gammaitoni, L., 2009. "Nonlinear energy harvesting". Physical Review Letters, 102(8), p. 080601.

[53] Erturk, A., Hoffmann, J., and Inman, D., 2009. "A piezomagnetoelastic structure for broadband vibration energy harvesting". Applied Physics Letters, 94(25), p. 254102.

[54] Barton, D. A., Burrow, S. G., and Clare, L. R., 2010. "Energy harvesting from vibrations with a nonlinear oscillator". Journal of Vibration and Acoustics, 132(2), p. 021009.

[55] Stanton, S. C., McGehee, C. C., and Mann, B. P., 2010. "Nonlinear dynamics for broadband energy harvesting: investigation of a bistable piezoelectric inertial generator". Physica D: Nonlinear Phenomena, 239(10), pp. 640-653.

[56] Sebald, G., Kuwano, H., Guyomar, D., and Ducharne, B., 2011. "Experimental duffing oscillator for broadband piezoelectric energy harvesting". Smart materials and structures, 20(10), p. 102001.

[57] Stanton, S. C., Owens, B. A., and Mann, B. P., 2012. "Harmonic balance analysis of the bistable piezoelectric inertial generator". Journal of Sound and Vibration, 331(15), pp. 3617-3627.

[58] Bilgen, O., Friswell, M. I., Ali, S. F., and Litak, G., 2015. "Broadband vibration energy harvesting from a vertical cantilever piezocomposite beam with tip mass". International Journal of Structural Stability and Dynamics, 15(02), p. 1450038.

[59] Zhou, S., Cao, J., Inman, D. J., Lin, J., Liu, S., and Wang, Z., 2014. "Broadband tristable energy harvester: Modeling and experiment verification". Applied Energy, 133, pp. 33-39.

[60] Gammaitoni, L., Neri, I., and Vocca, H., 2009. "Nonlinear oscillators for vibration energy harvesting". Applied Physics Letters, 94(16), p. 164102.

[61] Masana, R., and Daqaq, M. F., 2011. "Electromechanical modeling and nonlinear analysis of axially loaded energy harvesters". Journal of Vibration and Acoustics, 133(1), p. 011007.

[62] Marinkovic, B., and Koser, H., 2009. "Smart sanda wide bandwidth vibration energy harvesting platform". Applied Physics Letters, 94(10), p. 103505.

[63] Tvedt, L. G. W., Nguyen, D. S., and Halvorsen, E., 2010. "Nonlinear behavior of an electrostatic energy harvester under wide-and narrowband excitation". Microelectromechanical Systems, Journal of, 19(2), pp. 305-316.

[64] Miki, D., Honzumi, M., Suzuki, Y., and Kasagi, N., 2010. "Large-amplitude mems electret generator with nonlinear spring". In Micro Electro Mechanical Systems (MEMS), 2010 IEEE 23rd International Conference on, IEEE, pp. 176179.

[65] Nguyen, D., Halvorsen, E., Jensen, G., and Vogl, A., 2010. "Fabrication and characterization of a wideband mems energy harvester utilizing nonlinear springs". Journal of Micromechanics and Microengineering, 20(12), p. 125009.

[66] Erturk, A., and Inman, D., 2011. "Broadband piezoelectric power generation on high-energy orbits of the bistable duffing oscillator with electromechanical coupling". Journal of Sound and Vibration, 330(10), pp. $2339-2353$.

[67] Stanton, S. C., and Mann, B. P., 2009. "Harvesting energy from the nonlinear oscillations of a bistable piezoelectric inertial energy generator". In ASME 2009 International Design Engineering Technical Conferences and Computers and Information in Engineering Conference, American Society of Mechanical Engineers, pp. 447-456.

[68] Ferrari, M., Ferrari, V., Guizzetti, M., Andò, B., Baglio, S., and Trigona, C., 2009. "Improved energy harvesting from wideband vibrations by nonlinear piezoelectric converters". Procedia Chemistry, 1(1), pp. 1203-1206. 
Copyright [699] Ferrari, M., Ferrari, V., Guizzetti, M., Andò, B., Baglio, S., and Trigona, C., 2010. "Improved energy harvesting from wideband vibrations by nonlinear piezoelectric converters". Sensors and Actuators A: Physical, 162(2), pp. 425-431.

[70] Ferrari, M., Bau, M., Guizzetti, M., and Ferrari, V., 2011. "A single-magnet nonlinear piezoelectric converter for enhanced energy harvesting from random vibrations". Sensors and Actuators A: Physical, 172(1), pp. 287-292.

[71] Tang, L., WU, H., YANG, Y., and SOH, C. K., 2011. "Optimal performance of nonlinear energy harvesters". In 22nd International Conference on Adaptive Structures and Technologies.

[72] Tang, L., Yang, Y., and Soh, C.-K., 2012. "Improving functionality of vibration energy harvesters using magnets". Journal of Intelligent Material Systems and Structures, p. 1045389X12443016.

[73] Harne, R., Thota, M., and Wang, K., 2013. "Concise and high-fidelity predictive criteria for maximizing performance and robustness of bistable energy harvesters". Applied Physics Letters, 102(5), p. 053903.

[74] Stanton, S. C., Mann, B. P., and Owens, B. A., 2012. "Melnikov theoretic methods for characterizing the dynamics of the bistable piezoelectric inertial generator in complex spectral environments". Physica D: Nonlinear Phenomena, 241(6), pp. 711-720.

[75] Azizi, S., Chorsi, M. T., and Bakhtiari-nejad, F., 2016. "On the secondary resonance of a MEMS resonator : A conceptual study based on shooting and perturbation methods". International Journal of Non-Linear Mechanics, 82, pp. 59-68.

[76] Karami, M. A., and Inman, D. J., 2011. "Electromechanical modeling of the low-frequency zigzag micro-energy harvester". Journal of Intelligent Material Systems and Structures, 22(3), pp. 271-282.

[77] Arrieta, A., Delpero, T., Bergamini, A., and Ermanni, P., 2013. "Broadband vibration energy harvesting based on cantilevered piezoelectric bi-stable composites". Applied Physics Letters, 102(17), p. 173904.

[78] Gu, L., 2011. "Low-frequency piezoelectric energy harvesting prototype suitable for the mems implementation". Microelectronics Journal, 42(2), pp. 277-282.

[79] Karami, M. A., and Inman, D. J., 2011. "Equivalent damping and frequency change for linear and nonlinear hybrid vibrational energy harvesting systems". Journal of Sound and Vibration, 330(23), pp. 5583-5597.

[80] Tang, L., and Yang, Y., 2012. "A nonlinear piezoelectric energy harvester with magnetic oscillator". Applied Physics Letters, 101(9), p. 094102.

[81] Younis, M. I., 2011. MEMS linear and nonlinear statics and dynamics, Vol. 20. Springer Science \& Business Media. [82] Wolfram, S., 1987. Mathematica', June.

\section{Appendix: Coefficients of Magnetic Force}

This section lists the coefficients produced from the Taylor series expansion of the magnetic force:

$$
\begin{aligned}
& \alpha_{1}=\frac{F_{R}\left(d^{2}-4 Y_{s}^{2}\right)}{\left(d^{2}+Y_{s}^{2}\right)^{7 / 2} Y_{\phi}} \\
& \alpha_{2}=\frac{5 F_{R}\left(-3 d^{2}+4 Y_{s}^{2}\right)}{2\left(d^{2}+Y_{s}^{2}\right)^{9 / 2} Y_{\phi}^{2}} \\
& \alpha_{3}=\frac{5 F_{R}\left(d^{4}-12 d^{2} Y_{s}^{2}+8 Y_{s}^{4}\right)}{2\left(d^{2}+Y_{s}^{2}\right)^{11 / 2}} Y_{\phi}^{3} \\
& \alpha_{4}=\frac{35 F_{R} Y_{s}\left(5 d^{4}-20 d^{2} Y_{s}^{2}+8 Y_{s}^{4}\right)}{8\left(d^{2}+Y_{s}^{2}\right)^{13 / 2} Y_{\phi}^{4}} \\
& \alpha_{5}=\frac{7 F_{R}\left(5 d^{6}-120 d^{4} Y_{s}^{2}+240 d^{2} Y_{s}^{4}-64 Y_{s}^{6}\right)}{8\left(d^{2}+Y_{s}^{2}\right)^{15 / 2}} Y_{\phi}^{5} \\
& \alpha_{6}=\frac{21 F_{R} Y_{s}\left(-355 d^{6}+280 d^{4} Y_{s}^{2}-336 d^{2} Y_{s}^{4}+64 Y_{s}^{6}\right)}{16\left(d^{2}+Y_{s}^{2}\right)^{17 / 2} Y_{\phi}^{6}} \\
& \alpha_{7}=\frac{15 F_{R}\left(7 d^{8}-280 d^{6} Y_{s}^{2}+1120 d^{4} Y_{s}^{4}-896 d^{2} Y_{s}^{6}+128 Y_{s}^{8}\right)}{16\left(d^{2}+Y_{s}^{2}\right)^{19 / 2}} Y_{\phi}^{7} \\
& \alpha_{8}=\frac{165 F_{R} Y_{s}\left(63 d^{8}-840 d^{6} Y_{s}^{2}+2016 d^{4} Y_{s}^{4}-1152 d^{2} Y_{s}^{6}+128 Y_{s}^{8}\right)}{128\left(d^{2}+Y_{s}^{2}\right)^{21 / 2}} Y_{\phi}^{8} \\
& \alpha_{9}=\frac{55 F_{R}\left(21 d^{10}-1260 d^{8} Y_{s}^{2}-8400 d^{6} Y_{s}^{4}-13440 d^{4} Y_{s}^{6}-5760 d^{2} Y_{s}^{8}-512 Y_{s}^{10}\right)}{128\left(d^{2}+Y_{s}^{2}\right)^{23 / 2}} Y_{\phi}^{9}
\end{aligned}
$$




\section{Copyright Lisi of Figures}

1 Schematic of the experimental setup and the stability configurations of the resonator (a) Schematic of the experimental setup. (b) Mono-stable and bi-stable configurations. . . . . . . . . . . . . . . . .

2 The experimental setup of the piezoelectric EH, cantilever harvester to the right and PUMA Spectral Dy-

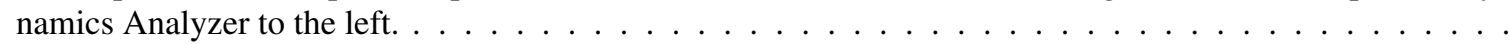

3 Schematic for the total magnetic force acting on the tip mass. . . . . . . . . . . . . . . . . .

4 The tip mass static response with the separation distance, $d$, between the two magnets. Threshold value, $d_{t h}$ found to be $20 \mathrm{~mm}$. Three values for mono-stable and bi-stable regions are selected for analysis. . . . . . .

5 First normalized mode shape plotted at different distances between the two magnets, where $d=20 \mathrm{~mm}$ is the threshold distance. . . . . . . . . . . . . . . . . . . . . . . . .

6 Variation of the first natural frequency with the distance, $d$, between the two magnets. Threshold value match static profile with value of $d_{t h}=20 \mathrm{~mm} \ldots \ldots \ldots \ldots \ldots$

7 Experimental and simulated forward sweep frequency and voltage responses for mono-stable resonator at $d=40 \mathrm{~mm}, A=0.5 \mathrm{~g}$ and damping $\mu=0.038$ (a) Tip mass displacement. (b) Output voltage. . . . . . . .

8 Experimental frequency forward swept responses for mono-stable resonator at $d=40 \mathrm{~mm}$ at different excitation levels (a) Tip mass displacement. (b) Output voltage. . . . . . . . . . . . . . . . . . . . . . .

9 Experimental frequency forward swept responses for mono-stable resonator at $d=22 \mathrm{~mm}$ at different excitation levels (a) Tip mass displacement. (b) Output voltage. . . . . . . . . . . . . . . . . . . . . . . .

10 Experimental frequency responses for bi-stable resonator at $d=5 \mathrm{~mm}$ (a) Tip mass displacement. (b) Output

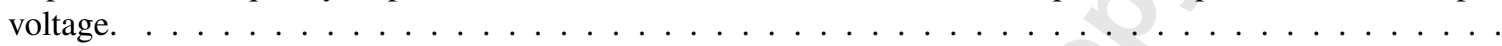

11 Experimental and simulated frequency responses for bi-stable resonator at $d=18 \mathrm{~mm}, A=0.5 \mathrm{~g}$ and damping $\mu=0.038$ (a) Tip mass displacement. (b) Output voltage. . . . . . . . . . . . . . . . . . . . .

12 Bi-stable simulated results for the nonlinear energy harvester at $d=18 \mathrm{~mm}, A=1.0 \mathrm{~g}$ and damping $\mu=0.038$ (a) Tip mass displacement. (b) Output voltage. . . . . . . . . . . . . . . . . . . . . . . . . .

13 The basin of attraction for the resonator for (a) $d=18 \mathrm{~mm}$ at $1.0 \mathrm{~g}, \Omega=18 \mathrm{~Hz}, \mu=0.038$. (b) $d=18 \mathrm{~mm}$ at $1.0 \mathrm{~g}$, fold frequency $\Omega=16.7 \mathrm{~Hz}, \mu=0.038$. Orange color is the basin of attraction for lower branch, while the black color is the basin of attraction for the upper branch. . . . . . . . . . . . .

14 Experimental frequency responses for bi-stable resonator at $d=18 \mathrm{~mm}$ :(a) Tip mass displacement. (b) Output voltage. . . . . . . . . . . . . . . . . . . . . . . . .

15 Simulated frequency responses with Shooting methods for bi-stable resonator at $d=18 \mathrm{~mm}$ : (a) Tip mass

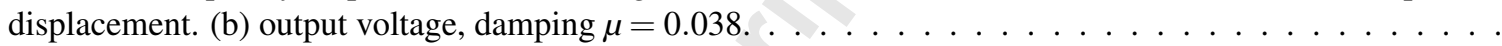

16 Simulated frequency responses with Long Time Integration and Shooting methods for bi-stable resonator at $d=18 \mathrm{~mm}$ : (a) Tip mass displacement. (b) Output voltage, damping $\mu=0.038$. . . . . . . . . . . . . .

17 Maximum output voltage with increasing the resistance up to $10 \mathrm{M} \Omega$ for an excitation level of $0.3 \mathrm{~g}$, separation distance of $18 \mathrm{~mm}$, and damping of $\mu=0.038$.

18 The bifurcation diagram of the actuator constructed from a force (excitation level) sweep at the threshold distance $\left(d_{t h}=20 \mathrm{~mm}\right)$ and its natural frequency of $\Omega=12.59 \mathrm{~Hz}$, single-sided Poincaré sections obtained at the period of excitation. . . . . . . . . . . . . . . . . . . . . .

19 Chaotic response for the harvester at $d_{t h}=20 \mathrm{~mm}, \Omega=12.59 \mathrm{~Hz}$, and $0.2228 \mathrm{~g}$ excitation level:(a) Phase

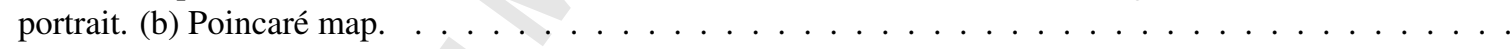

20 The bifurcation diagram sweeping the frequency of excitation at $d_{t h}=20 \mathrm{~mm}$ and $A m p=0.2 \mathrm{~g}$, single-sided Poincaré sections obtained at the period of excitation. . . . . . . . . . . . . . . . .

21 Maximum output voltage as the distance between magnets varies at the excitation level of $1.0 \mathrm{~g}$. (b) Dynamics behavior for energy harvester. . . . . . . . . . . . . . . . . . . . . . 


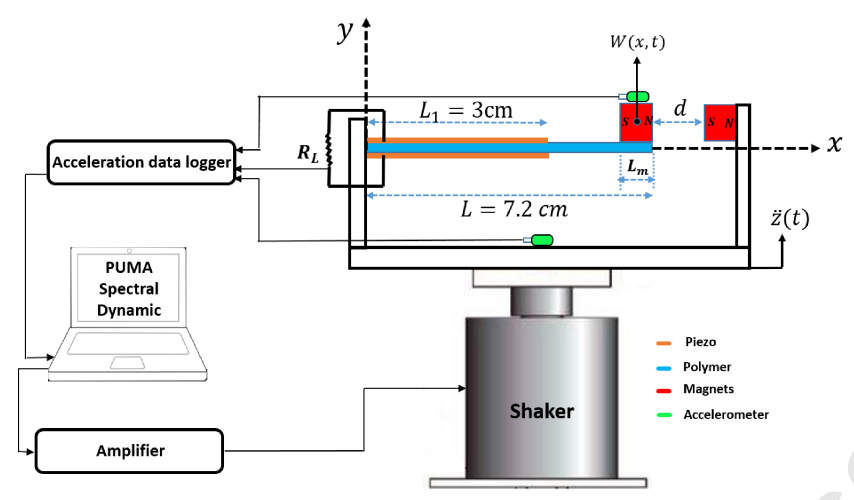

(a)

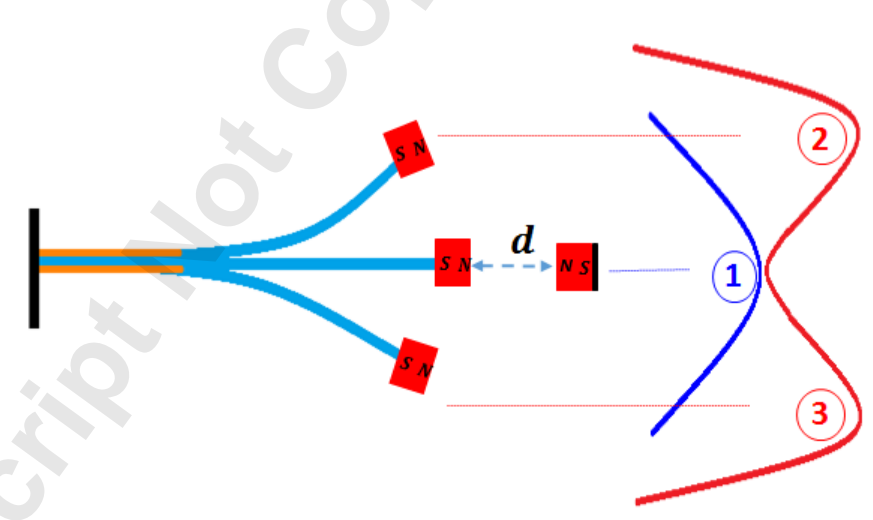

(b)

Fig. 1: Schematic of the experimental setup and the stability configurations of the resonator (a) Schematic of the experimental setup. (b) Mono-stable and bi-stable configurations. 


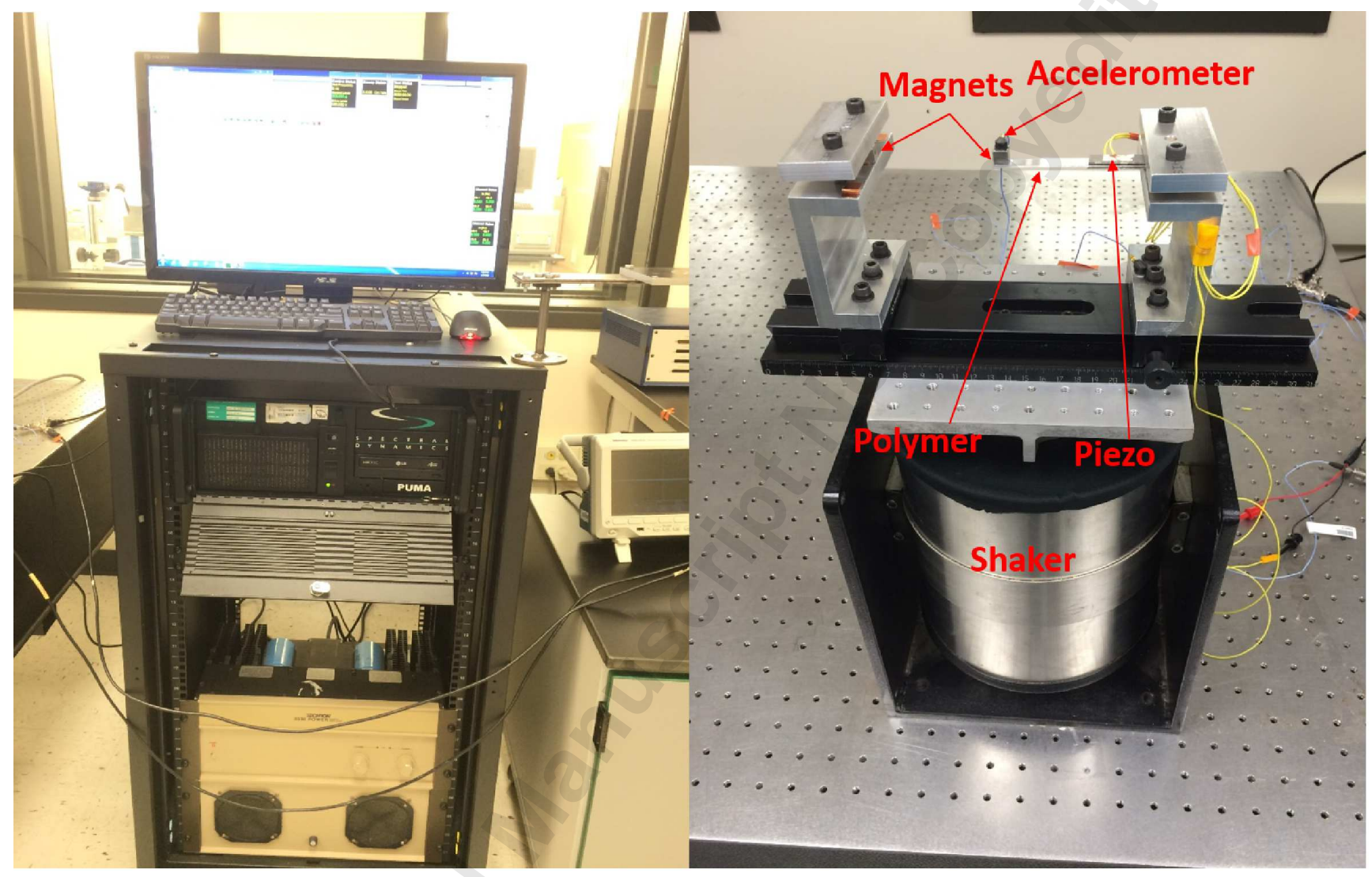

Fig. 2: The experimental setup of the piezoelectric EH, cantilever harvester to the right and PUMA Spectral Dynamics Analyzer to the left. 


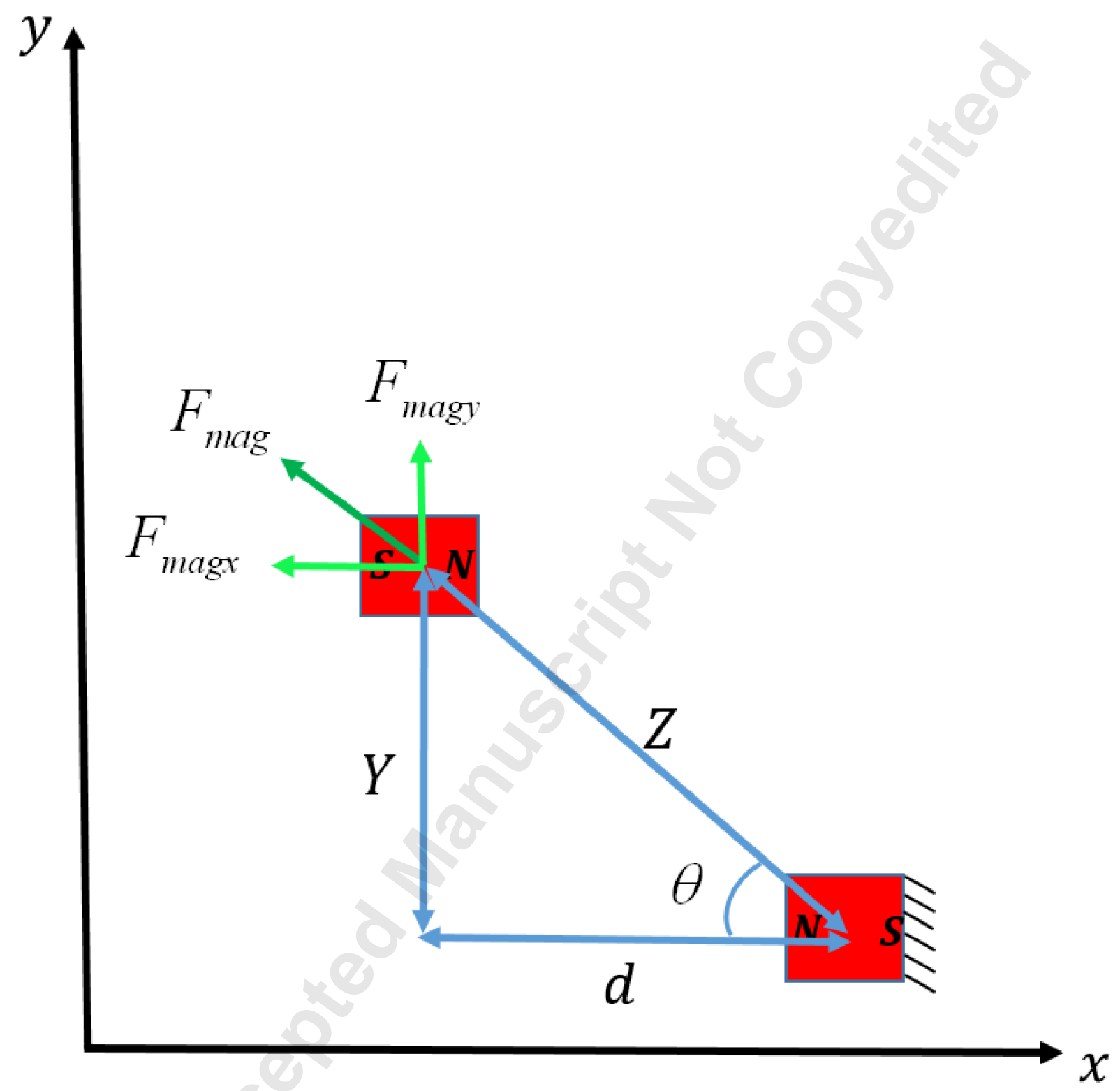

Fig. 3: Schematic for the total magnetic force acting on the tip mass. 


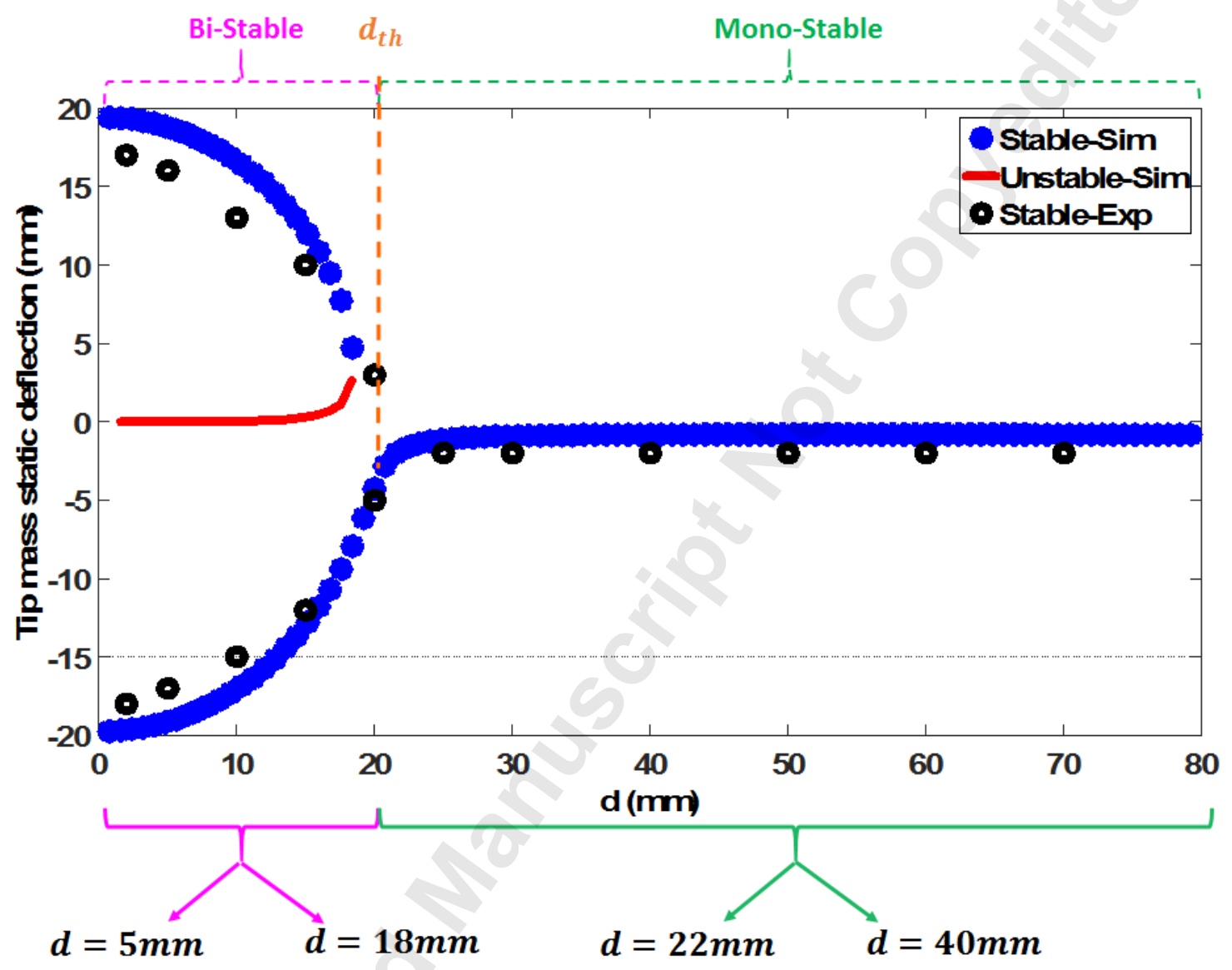

Fig. 4: The tip mass static response with the separation distance, $d$, between the two magnets. Threshold value, $d_{t h}$ found to be $20 \mathrm{~mm}$. Three values for mono-stable and bi-stable regions are selected for analysis. 


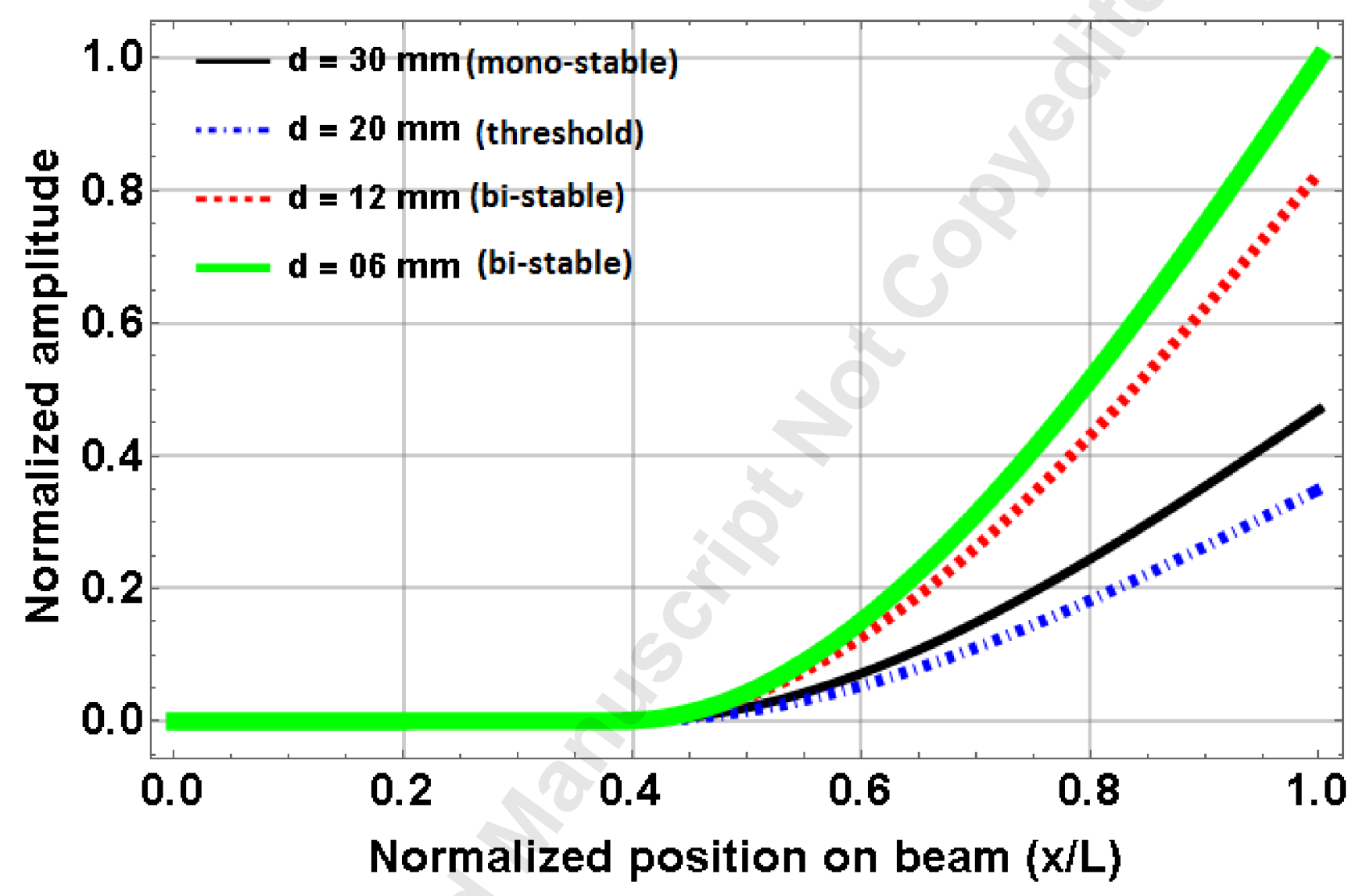

Fig. 5: First normalized mode shape plotted at different distances between the two magnets, where $d=20 \mathrm{~mm}$ is the threshold distance. 


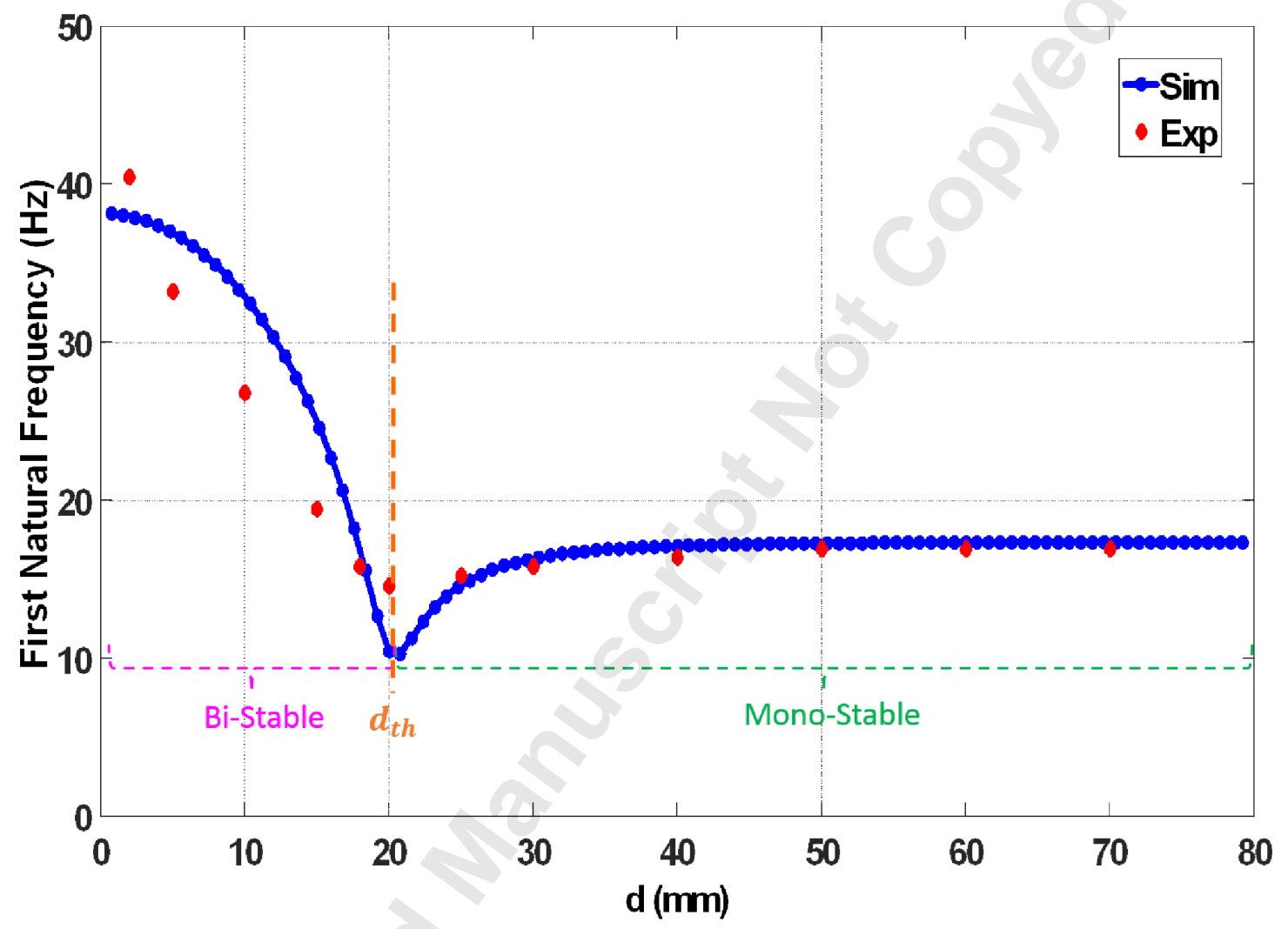

Fig. 6: Variation of the first natural frequency with the distance, $d$, between the two magnets. Threshold value match static profile with value of $d_{t h}=20 \mathrm{~mm}$. 


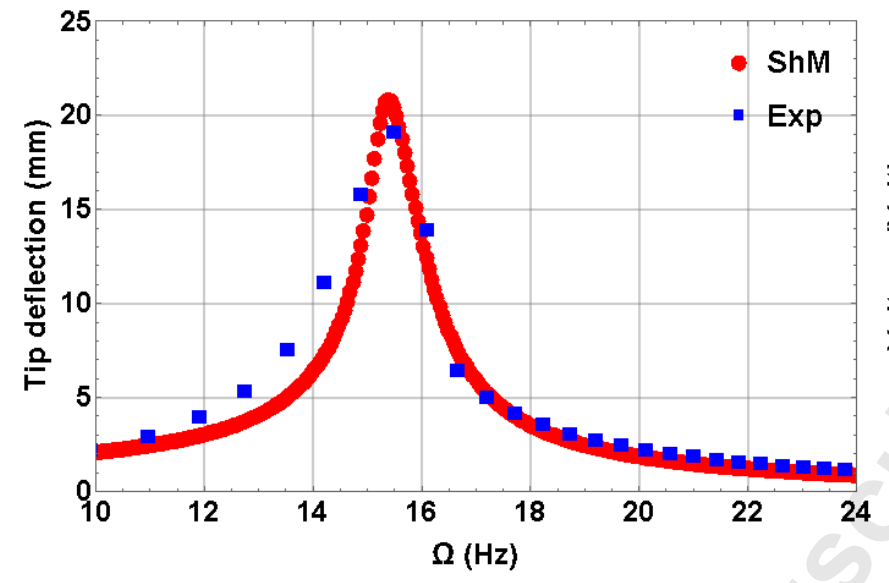

(a)

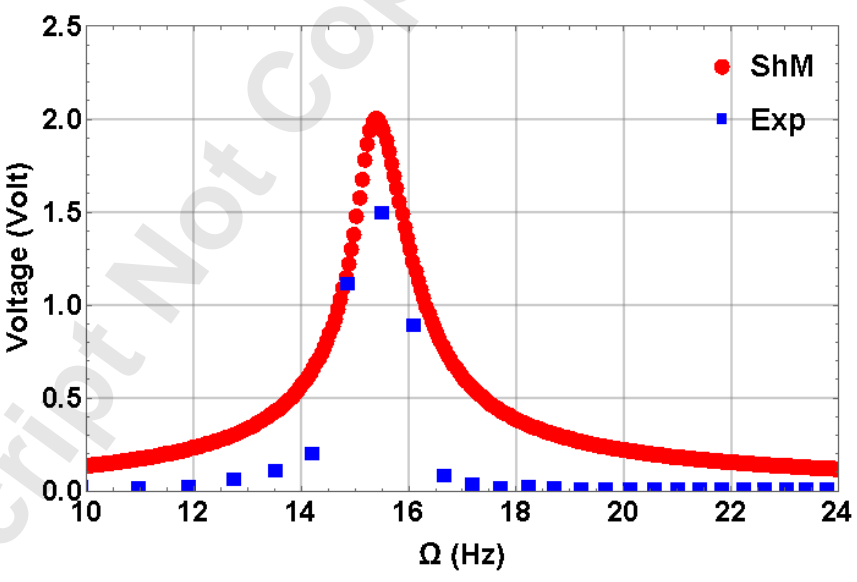

(b)

Fig. 7: Experimental and simulated forward sweep frequency and voltage responses for mono-stable resonator at $d=40 \mathrm{~mm}$, $A=0.5 \mathrm{~g}$ and damping $\mu=0.038$ (a) Tip mass displacement. (b) Output voltage. 


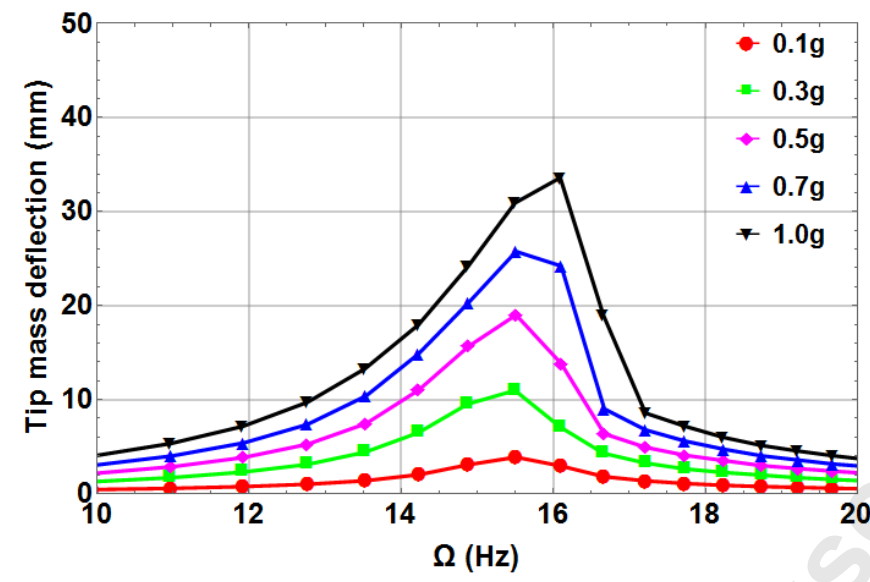

(a)

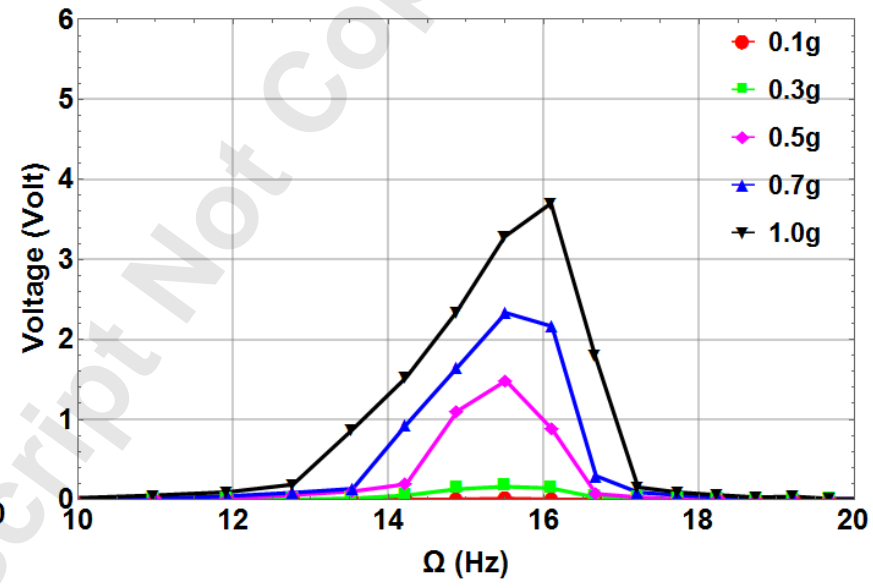

(b)

Fig. 8: Experimental frequency forward swept responses for mono-stable resonator at $d=40 \mathrm{~mm}$ at different excitation levels (a) Tip mass displacement. (b) Output voltage. 


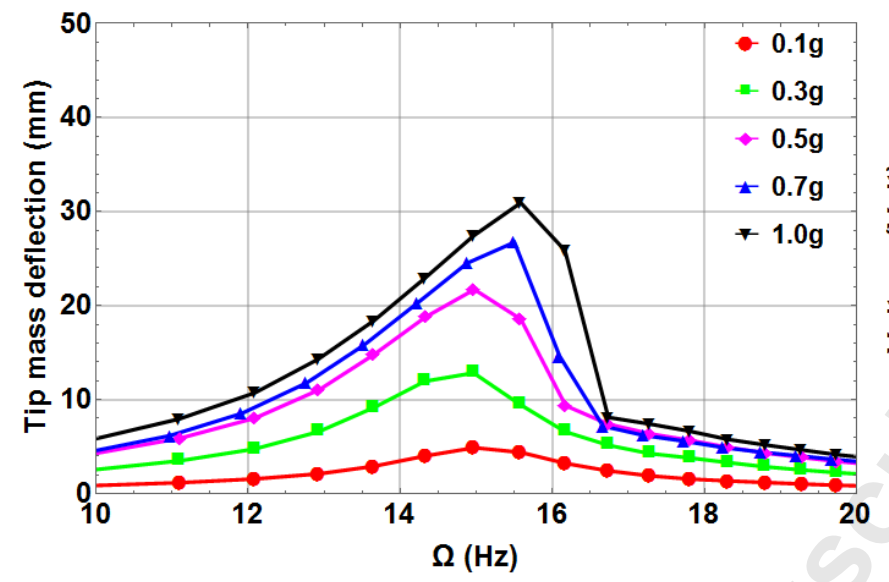

(a)

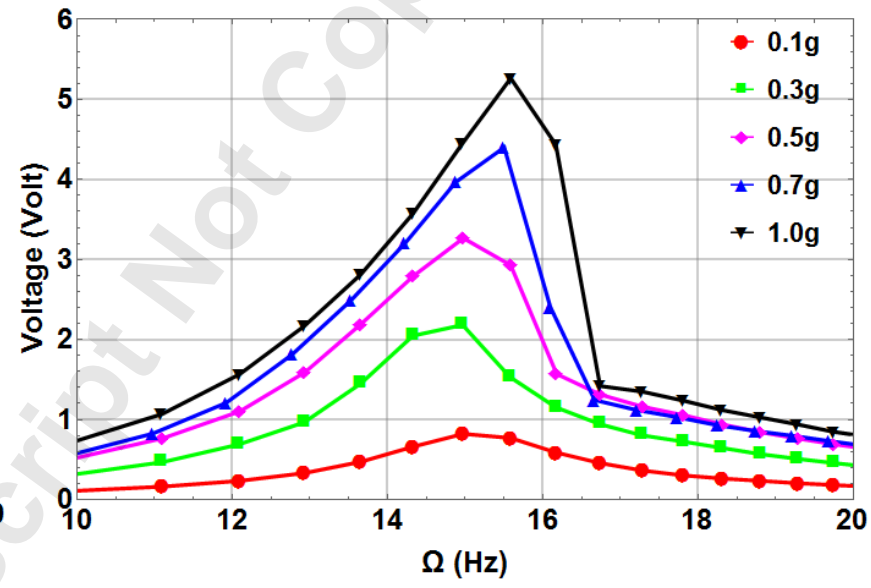

(b)

Fig. 9: Experimental frequency forward swept responses for mono-stable resonator at $d=22 \mathrm{~mm}$ at different excitation levels (a) Tip mass displacement. (b) Output voltage. 


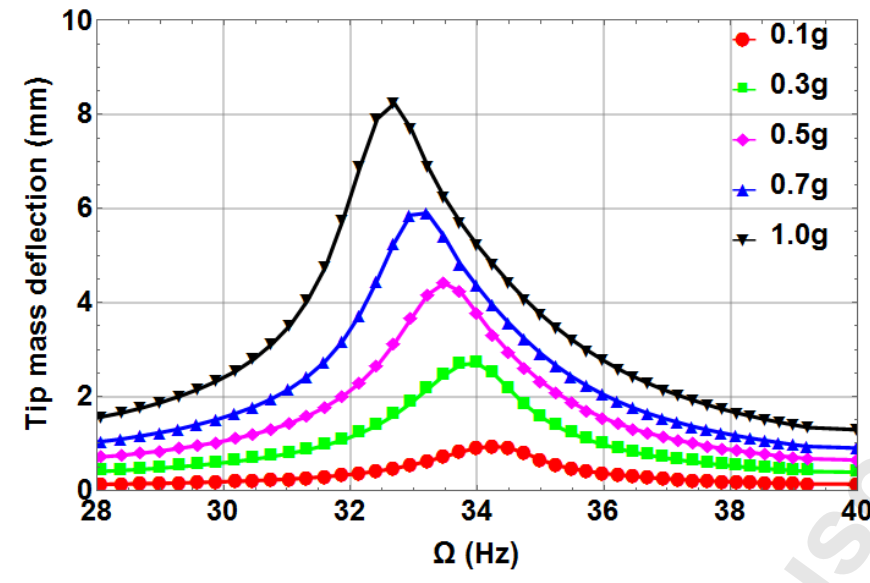

(a)

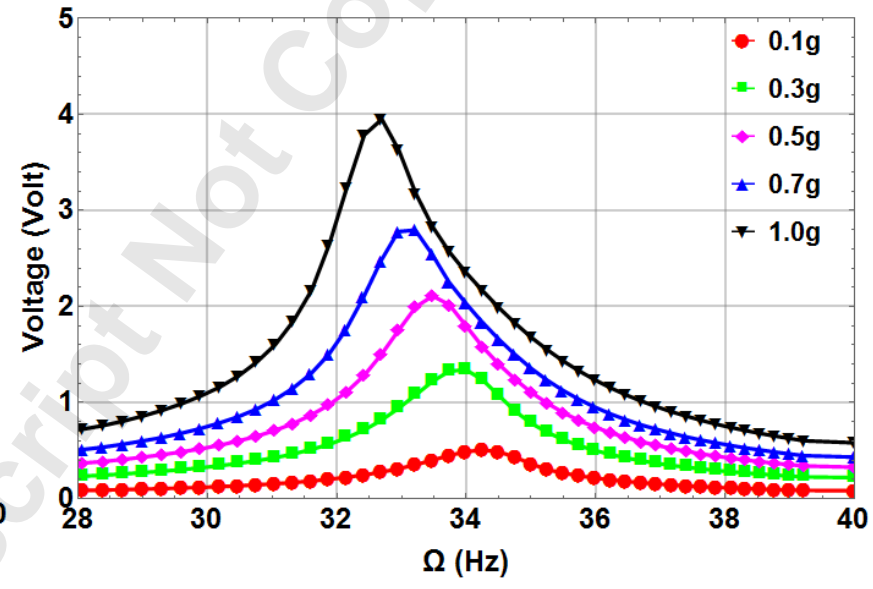

(b)

Fig. 10: Experimental frequency responses for bi-stable resonator at $d=5 \mathrm{~mm}$ (a) Tip mass displacement. (b) Output voltage. 


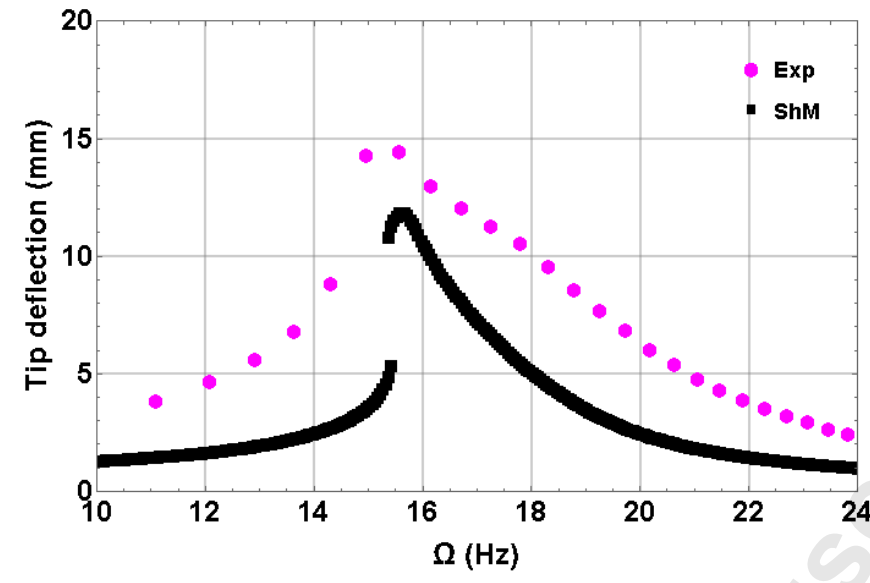

(a)

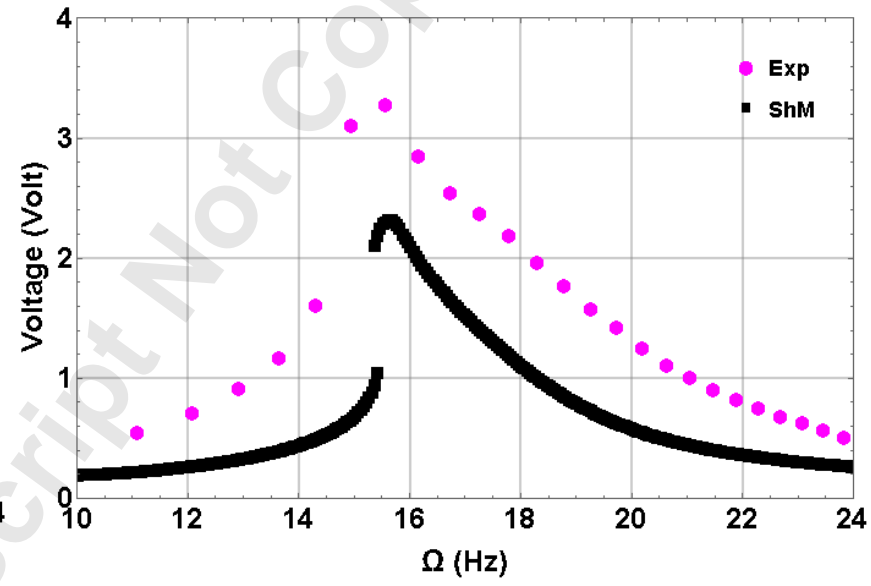

(b)

Fig. 11: Experimental and simulated frequency responses for bi-stable resonator at $d=18 \mathrm{~mm}, A=0.5 \mathrm{~g}$ and damping $\mu=0.038$ (a) Tip mass displacement. (b) Output voltage. 


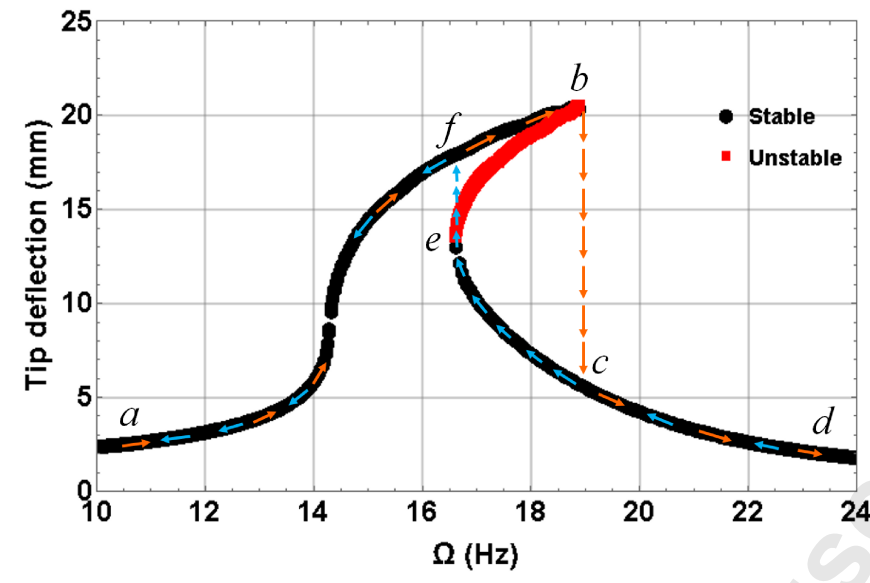

(a)

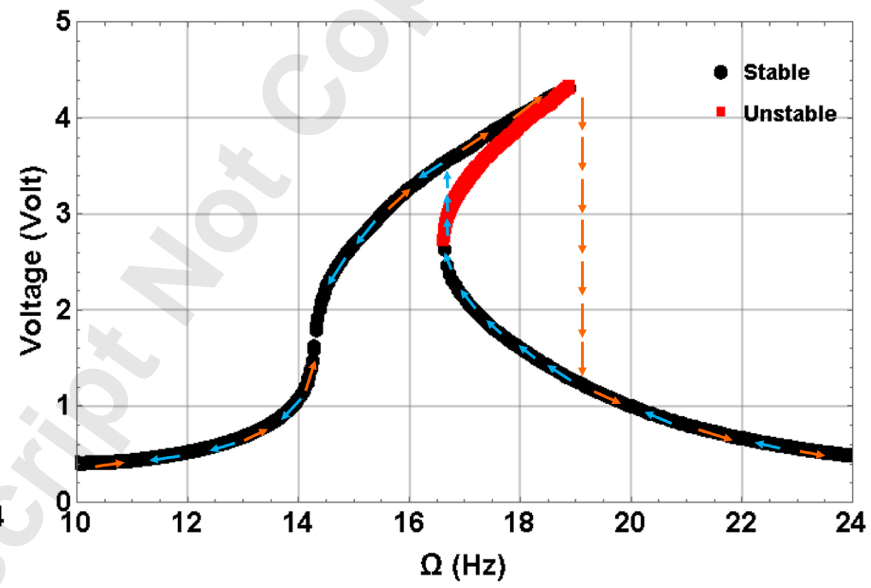

(b)

Fig. 12: Bi-stable simulated results for the nonlinear energy harvester at $d=18 \mathrm{~mm}, A=1.0 \mathrm{~g}$ and damping $\mu=0.038$ (a) Tip mass displacement. (b) Output voltage. 


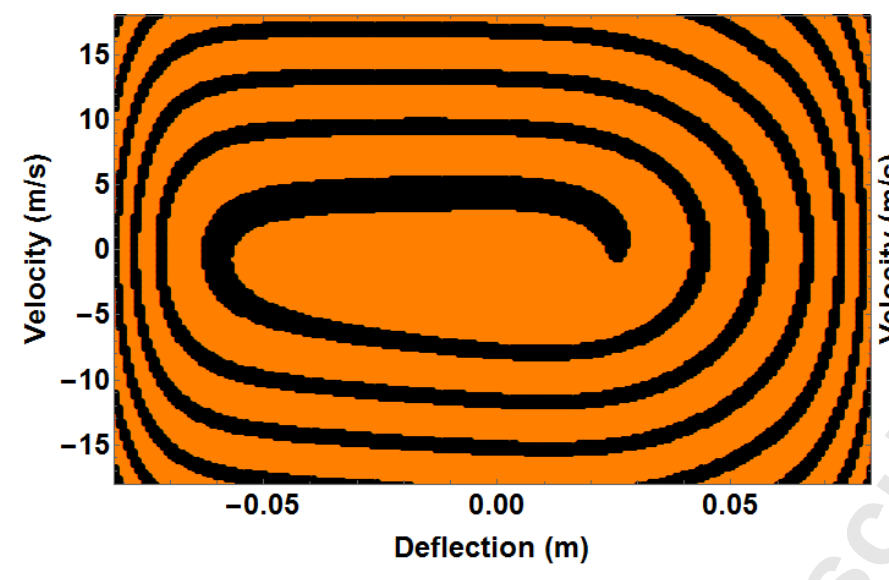

(a)

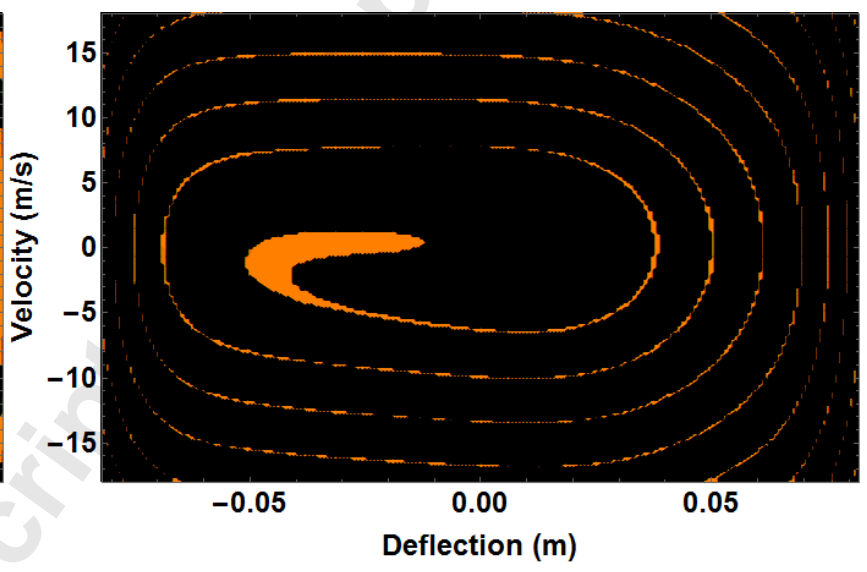

(b)

Fig. 13: The basin of attraction for the resonator for (a) $d=18 \mathrm{~mm}$ at $1.0 \mathrm{~g}, \Omega=18 \mathrm{~Hz}, \mu=0.038$. (b) $\mathrm{d}=18 \mathrm{~mm}$ at $1.0 \mathrm{~g}$, fold frequency $\Omega=16.7 \mathrm{~Hz}, \mu=0.038$. Orange color is the basin of attraction for lower branch, while the black color is the basin of attraction for the upper branch. 


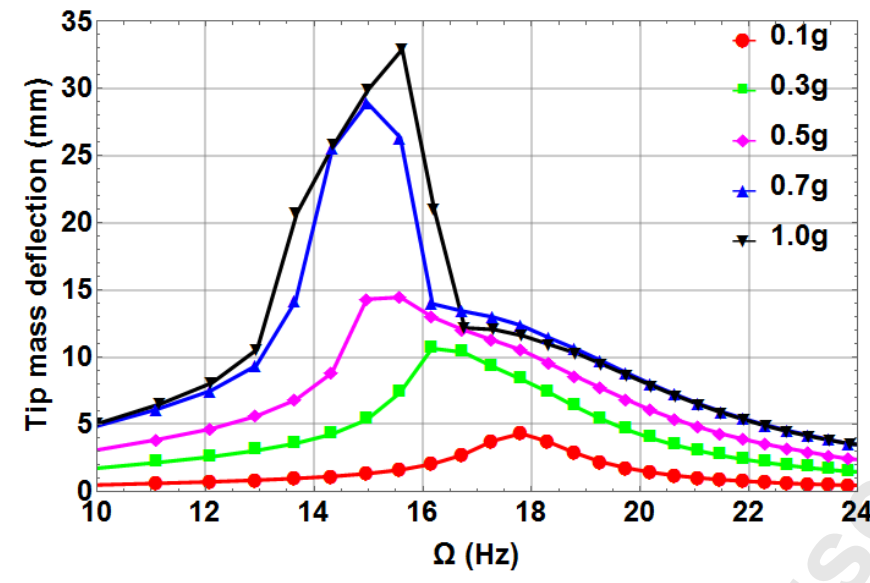

(a)

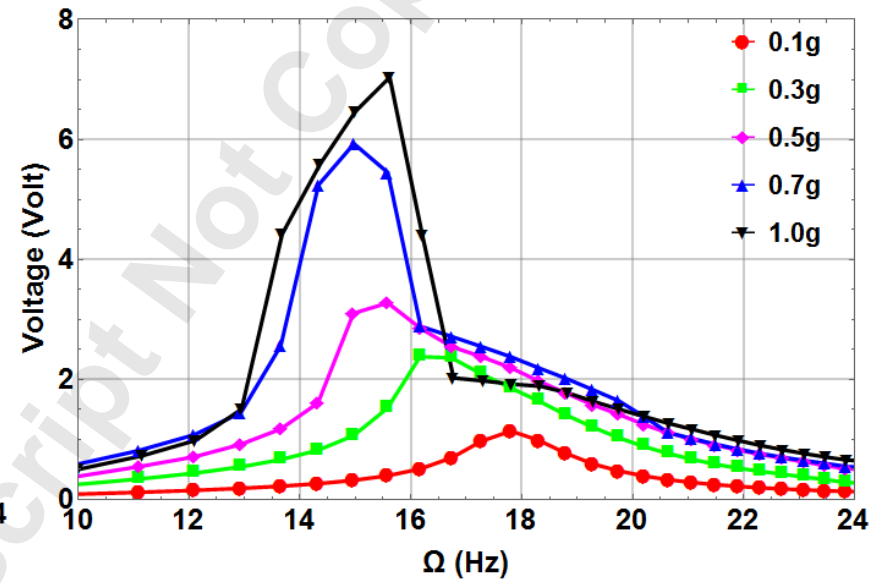

(b)

Fig. 14: Experimental frequency responses for bi-stable resonator at $d=18 \mathrm{~mm}$ :(a) Tip mass displacement. (b) Output voltage. 


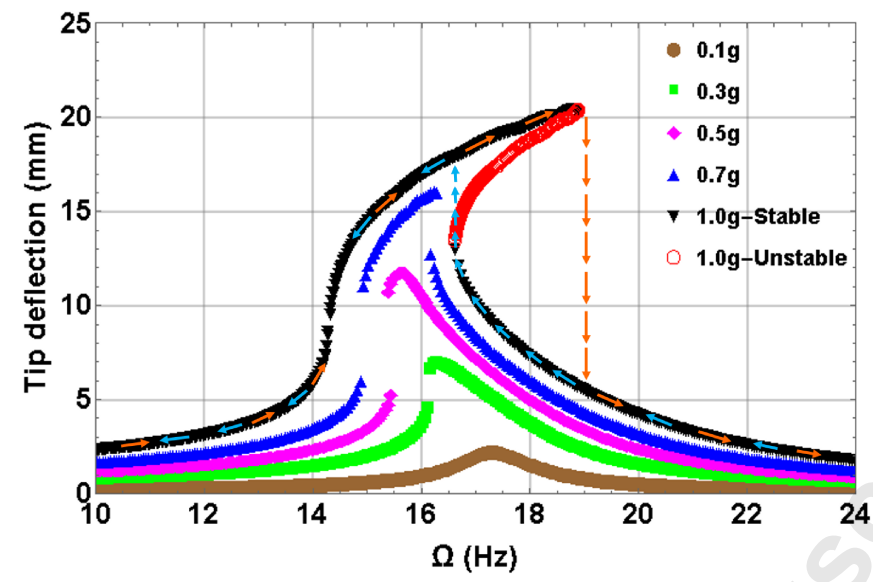

(a)

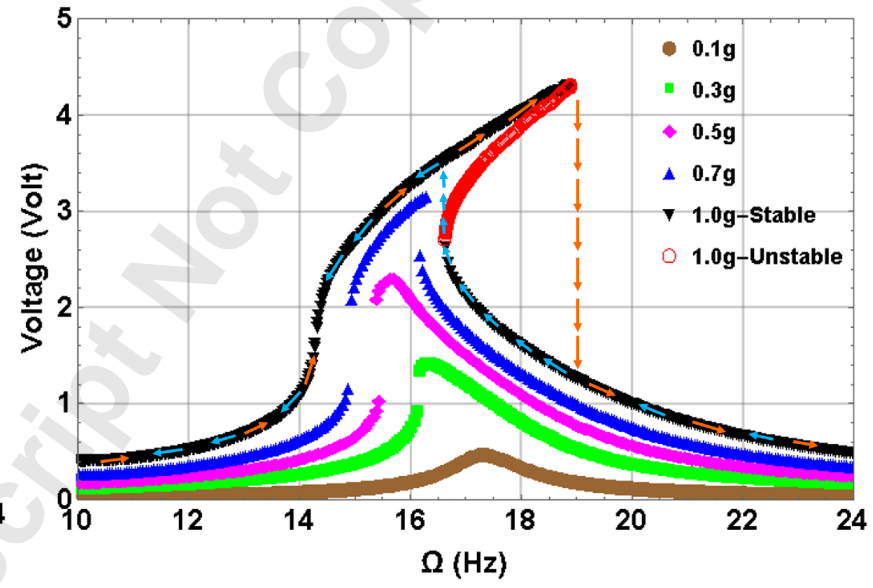

(b)

Fig. 15: Simulated frequency responses with Shooting methods for bi-stable resonator at $d=18 \mathrm{~mm}$ : (a) Tip mass displacement. (b) output voltage, damping $\mu=0.038$. 


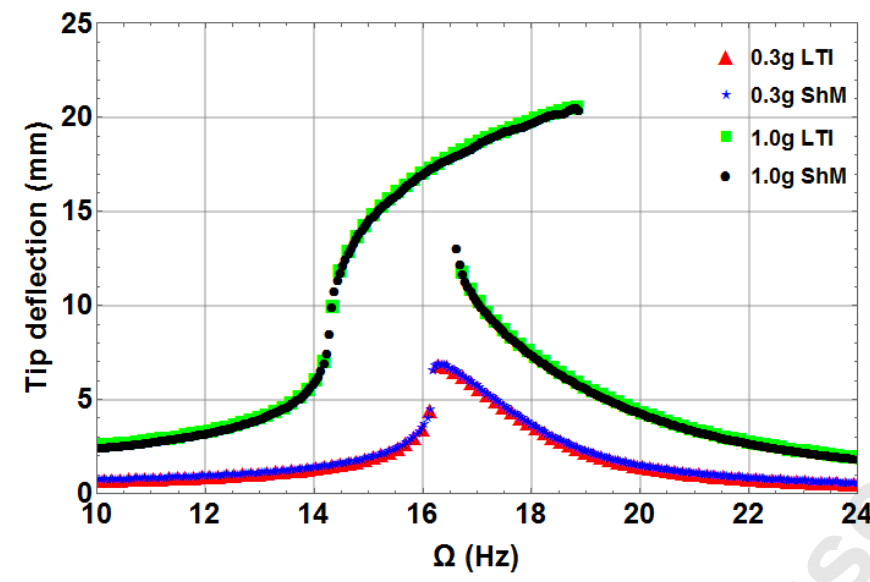

(a)

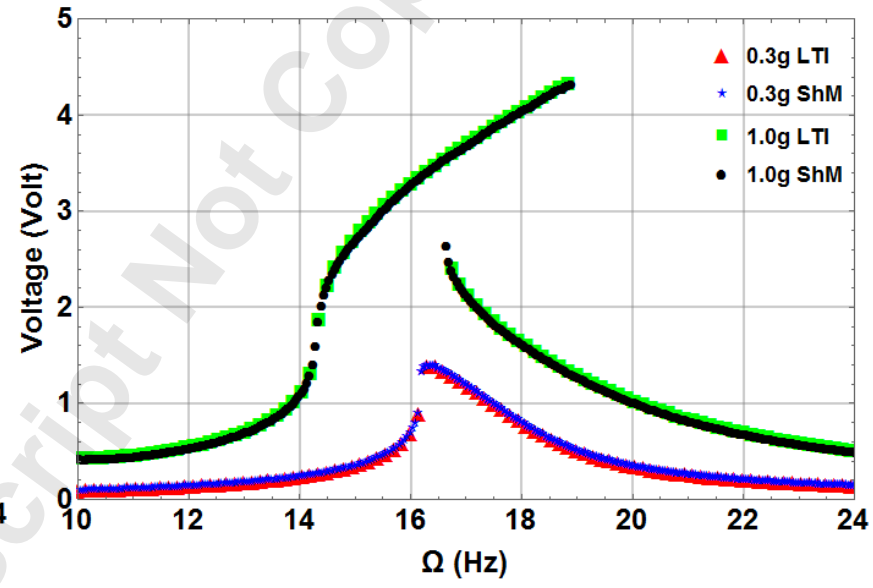

(b)

Fig. 16: Simulated frequency responses with Long Time Integration and Shooting methods for bi-stable resonator at $d=$ $18 \mathrm{~mm}$ : (a) Tip mass displacement. (b) Output voltage, damping $\mu=0.038$. 


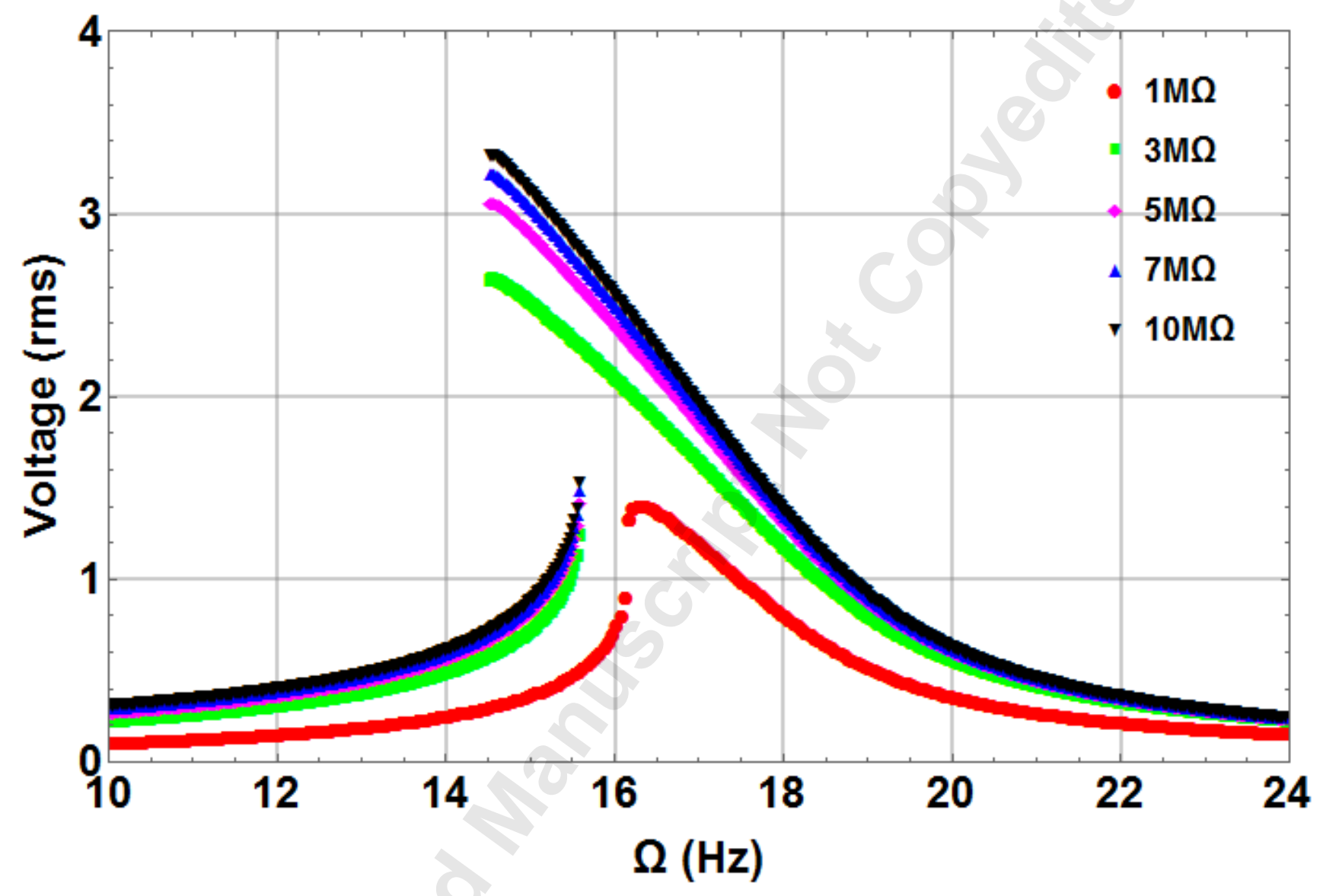

Fig. 17: Maximum output voltage with increasing the resistance up to $10 M \Omega$ for an excitation level of $0.3 \mathrm{~g}$, separation distance of $18 \mathrm{~mm}$, and damping of $\mu=0.038$. 


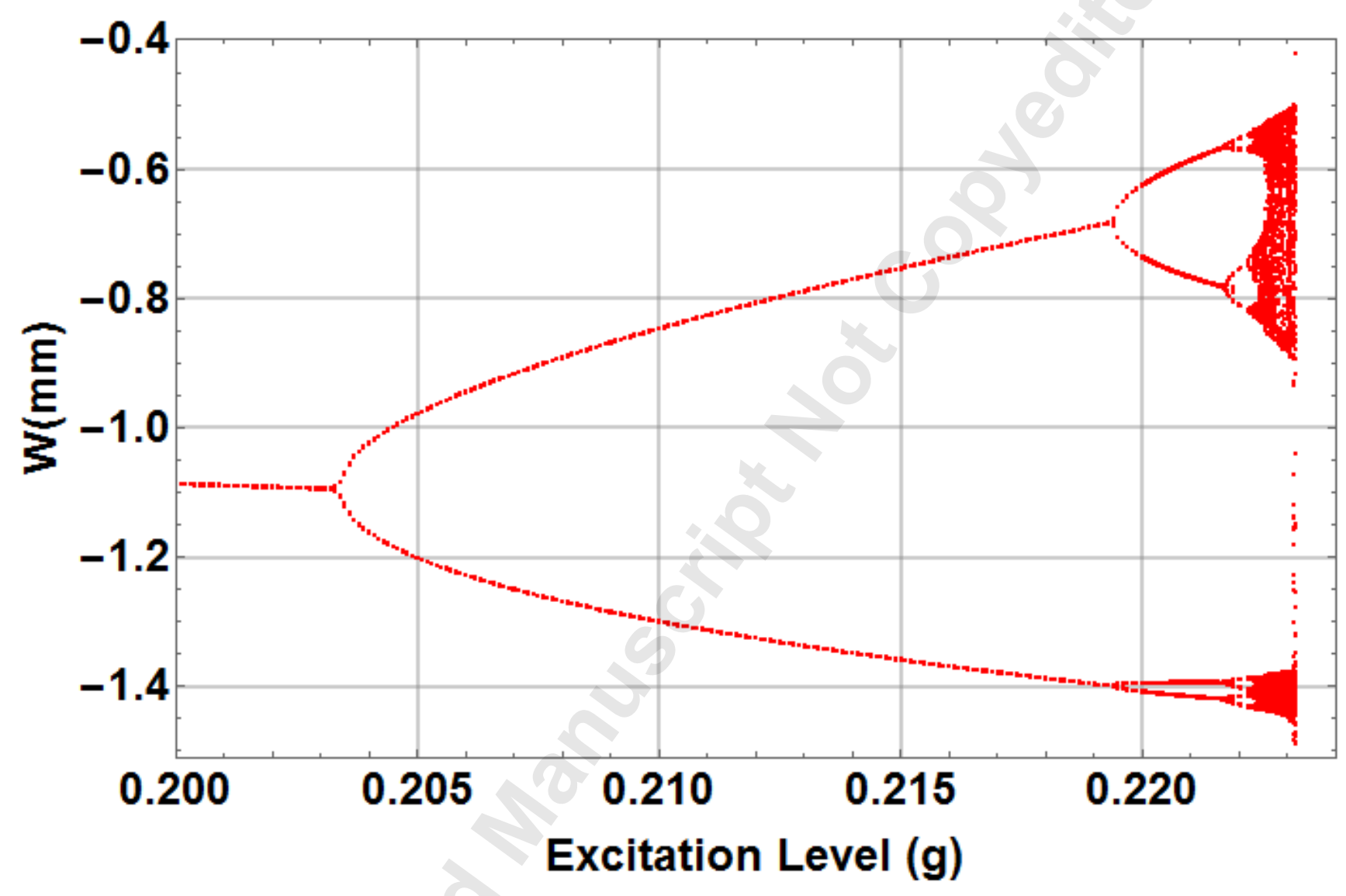

Fig. 18: The bifurcation diagram of the actuator constructed from a force (excitation level) sweep at the threshold distance $\left(d_{t h}=20 \mathrm{~mm}\right)$ and its natural frequency of $\Omega=12.59 \mathrm{~Hz}$, single-sided Poincaré sections obtained at the period of excitation. 


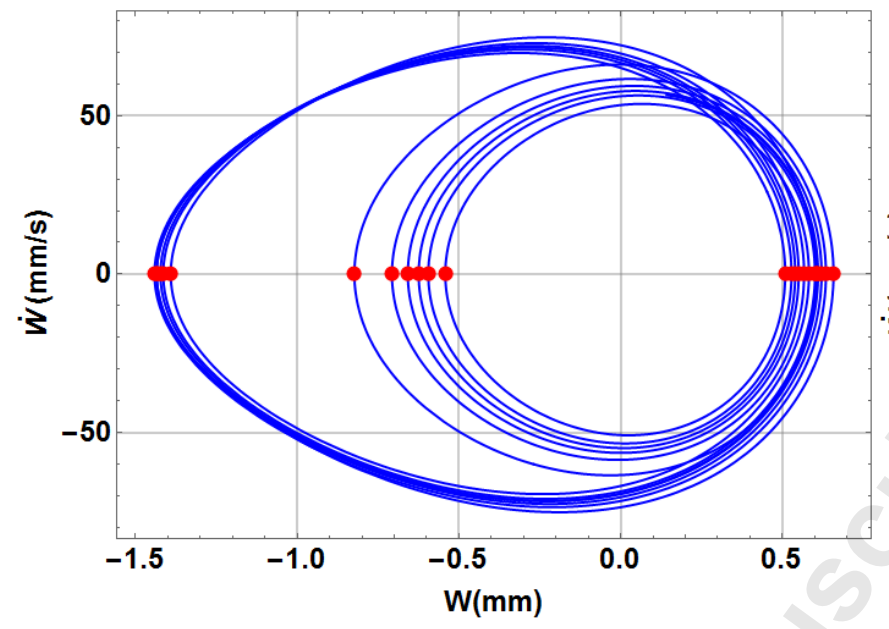

(a)

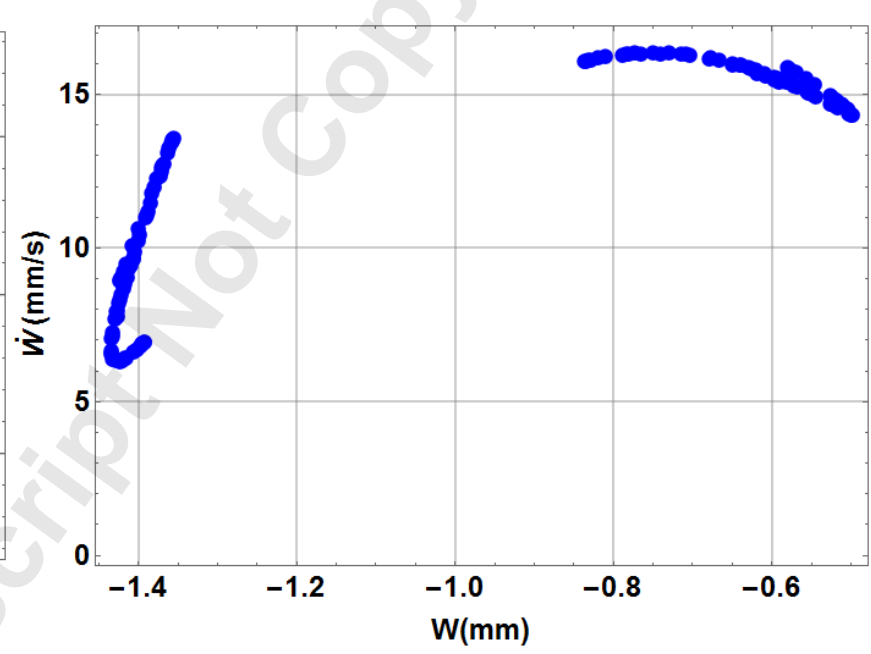

(b)

Fig. 19: Chaotic response for the harvester at $d_{t h}=20 \mathrm{~mm}, \Omega=12.59 \mathrm{~Hz}$, and $0.2228 \mathrm{~g}$ excitation level:(a) Phase portrait. (b) Poincaré map. 


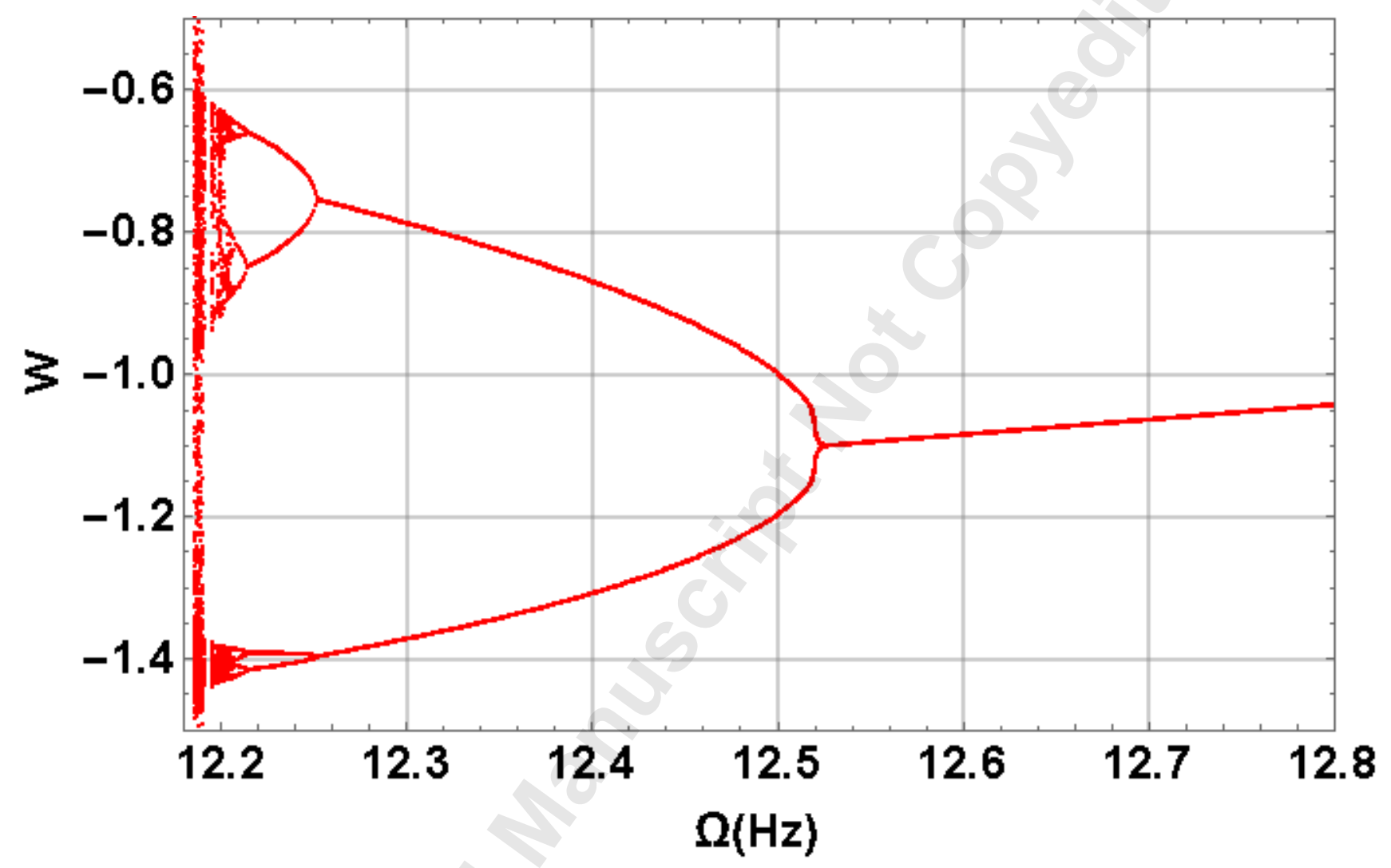

Fig. 20: The bifurcation diagram sweeping the frequency of excitation at $d_{t h}=20 \mathrm{~mm}$ and $A m p=0.2 \mathrm{~g}$, single-sided Poincaré sections obtained at the period of excitation. 


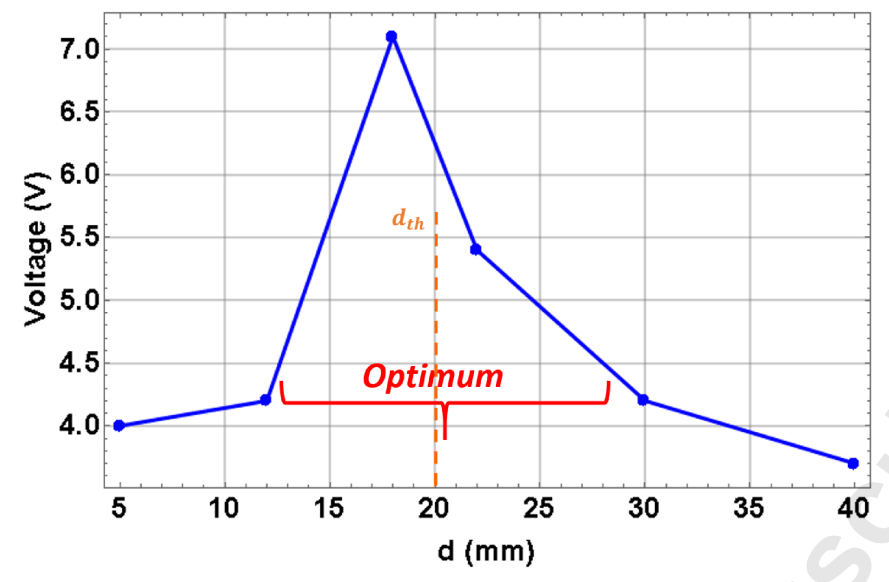

(a)

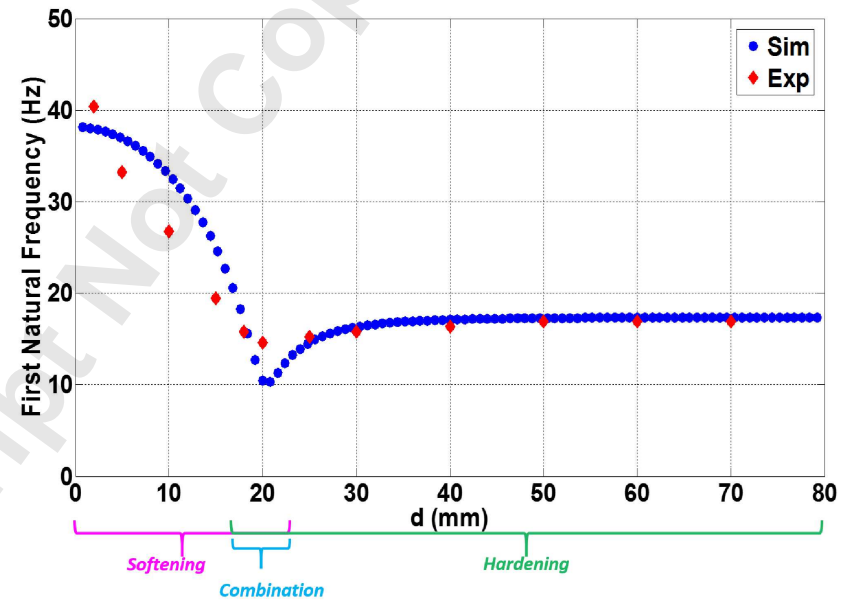

(b)

Fig. 21: Maximum output voltage as the distance between magnets varies at the excitation level of $1.0 \mathrm{~g}$. (b) Dynamics behavior for energy harvester. 
Journal of Vibration and Acoustics. Received September 09, 2016;

Accepted manuscript posted April 20, 2017. doi:10.1115/1.4036503

Copyright Lisi or Tables

1 Geometrical and material properties of the harvester.

VIB-16-1454 . Towfighian . 39

Downloaded From: http://vibrationacoustics.asmedigitalcollection.asme.org/pdfaccess.ashx?url=/data/journals/jvacek/0/ on 04/26/2017 Terms of Use: http://www.asme.org/abou 
Table 1: Geometrical and material properties of the harvester.

\begin{tabular}{llll}
\hline Parameters & Polymer & PZR 5A & Magnet \\
\hline Length $(\mathrm{cm})$ & 7.2 & 3 & 0.8 \\
Width $(\mathrm{cm})$ & 1.0 & 1.0 & 0.8 \\
Thickness $(\mathrm{cm})$ & 0.1 & 0.1 & 0.8 \\
Density $\left(\mathrm{kg} / \mathrm{m}^{3}\right)$ & 1220 & 7800 & 7500 \\
Modulus $(\mathrm{GPa})$ & 2.344 & 66 & - \\
Magnetic moment $\left(\mathrm{A}^{2} / \mathrm{m}\right)$ & - & - & 0.5 \\
Piezoelectric constant & - & -3 & -
\end{tabular}

VIB-16-1454 . Towfighian . 40 